\title{
AN OLD NEW CLASS OF MEROMORPHIC FUNCTIONS
}

\author{
NORBERT STEINMETZ
}

\section{Abstract}

Based on the so-called re-scaling method, we will give a detailed description of the solutions to the Hamiltonian system (1) below, which was discovered only recently by Kecker [4, 5], and is strongly related to Painlevé's fourth differential equation. In particular, the problem to determine those fourth Painlevé transcendents with positive Nevanlinna deficiency $\delta(0, w)$, is completely resolved.

Keywords. Hamiltonian system, Riccati differential equation, Painlevé transcendent, asymptotic series, re-scaling, pole-free sector, Nevanlinna deficiency

2010 MSC. 30D30, 30D35, 30D45

\section{INTRODUCTION}

The Hamiltonian system

$$
p^{\prime}=-q^{2}-z p-\alpha, q^{\prime}=p^{2}+z q+\beta
$$

with time-dependent Hamiltonian

$$
H(z, p, q)=\frac{1}{3}\left(p^{3}+q^{3}\right)+z p q+\beta p+\alpha q
$$

has been discovered by Kecker [4, 5] when questing for systems having the so-called (pseudo) Painlevé property. Roughly speaking, this means that the system has "no movable singularities but poles", or, more precisely, that every solution admits unrestricted analytic continuation in the plane except for fixed singularities (which do not occur here); and "pseudo" means that, in addition, also moving algebraic singularities are admitted. It is not hard to verify, although more elaborate to discover, that $w=p+q-z$ solves the differential equation

$$
2 w w^{\prime \prime}=w^{2}-w^{4}-4 z w^{3}-\left(2 \alpha+2 \beta+3 z^{2}\right) w^{2}-(\alpha-\beta+1)^{2} .
$$

Kecker [4] found some implicit second order equation for $q$. Obviously, equation (3) is closely related to Painlevé's fourth equation

$$
2 y y^{\prime \prime}=y^{\prime 2}+3 y^{4}+8 z y^{3}+4\left(z^{2}-\hat{\alpha}\right) y^{2}+2 \hat{\beta}
$$

with parameters $\hat{\alpha}=\frac{i}{\sqrt{3}}(\alpha+\beta)$ and $\hat{\beta}=\frac{2}{9}(\alpha-\beta+1)^{2}$; one has just to consider $y(z)=a w(b z)$ with $b=\sqrt[4]{-\frac{4}{3}}$ and $a=-\frac{1}{2} b^{3}$. Since also $\tilde{w}=\omega p+\bar{\omega} q-z\left(\omega^{3}=1\right)$ is a solution to (3) with $(\alpha, \beta)$ replaced by $(\omega \alpha, \bar{\omega} \beta), p$ and $q$ may be re-discovered:

$$
(\omega-\bar{\omega}) p=\tilde{w}-\bar{\omega} w-(\bar{\omega}-1) z \quad \text { and } \quad(\bar{\omega}-\omega) q=\tilde{w}-\omega w-(\omega-1) z .
$$

This could be the end of the story. The "old new" transcendents $p$ and $q$, however, have so many interesting properties that it seems justified to study them in their own right. 


\section{NotATION AND SIMPLE PROPERTIES}

By $\Lambda$ we denote the set of non-zero poles of $p$. It is easily seen that the poles $\lambda$ are simple, and $p$ and $q$ have residues $\varrho$ and $-\bar{\varrho}$, respectively, where $\varrho$ is some third root of unity. Thus $\Lambda$ is divided in a natural way into three disjoint sets $\Lambda^{\varrho}, \varrho^{3}=1$. With $\Lambda$ we associate the sets

$$
\triangle_{\delta}(\lambda)=\left\{z:|z-\lambda|<\delta|\lambda|^{-1}\right\} \quad \text { and } \quad \Lambda_{\delta}=\bigcup_{\lambda \in \Lambda} \triangle_{\delta}(\lambda) .
$$

If it is clear which solution $(p, q)$ is under consideration, we will also write $H(z)=$ $H(z, p(z), q(z))$. The following holds at poles in $\Lambda^{1}$ :

$$
\begin{gathered}
p(z)=\quad(z-\lambda)^{-1}+\frac{1}{2} \lambda+\left(1+\frac{1}{3}(\alpha-2 \beta)-\frac{1}{4} \lambda^{2}\right)(z-\lambda) \\
\quad+\left(\mathbf{h}-\left(\frac{5}{8}+\frac{1}{4}(\alpha-\beta)\right) \lambda\right)(z-\lambda)^{2}+\cdots \\
q(z)=(z-\lambda)^{-1}+\frac{1}{2} \lambda+\left(1+\frac{1}{3}(2 \alpha-\beta)+\frac{1}{4} \lambda^{2}\right)(z-\lambda) \\
\quad+\left(\mathbf{h}+\left(\frac{5}{8}+\frac{1}{4}(\alpha-\beta)\right) \lambda\right)(z-\lambda)^{2}+\cdots \\
H(z)=(z-\lambda)^{-1}+\left[2 \mathbf{h}+\frac{1}{3} \lambda^{3}+\frac{1}{2}(\alpha+\beta) \lambda\right]+\left[\frac{1}{3}(\alpha+\beta)+\frac{3}{4} \lambda^{2}\right](z-\lambda)+\cdots \\
p(z)+q(z)-z=(1+\alpha-\beta)(z-\lambda)+2 \mathbf{h}(z-\lambda)^{2}+\cdots
\end{gathered}
$$

The coefficient $\mathbf{h}=\mathbf{h}(\lambda)$ remains undetermined and free. Actually $\lambda$ and $\mathbf{h}$ may be prescribed to determine a unique solution. To determine the Laurent series at poles with residue $\varrho\left(\varrho^{3}=1\right)$, we replace $p$ and $q$ by $x=\bar{\varrho} p$ and $y=\varrho q$, respectively. Then $x$ and $y$ satisfy $x^{\prime}=-y^{2}-z x-\varrho \alpha, y^{\prime}=x^{2}+z y+\varrho \beta$, and we thus obtain the Laurent developments for $p=\varrho x$ and $q=\varrho y$ by replacing $(p, q, \alpha, \beta)$ in (7) by $(x, y, \bar{\varrho} \alpha, \varrho \beta)$ :

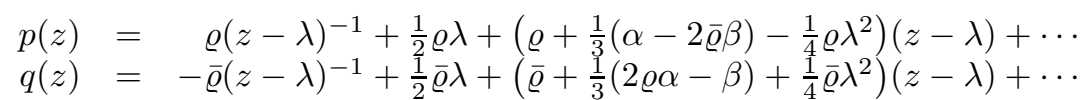

In particular, this means that the development (8) remains valid at poles with residue $\varrho$, provided $\alpha$ and $\beta$ are replaced by $\varrho \alpha$ and $\varrho \beta$, respectively. Hence $g(z)=$ $\exp \left(\int H(z) d z\right)$ is an entire function that has simple zeros at the poles of $p$, and no others.

2.1. Bäcklund transformations. Trivial Bäcklund transformations are $\left(\omega^{3}=1\right.$ arbitrary)

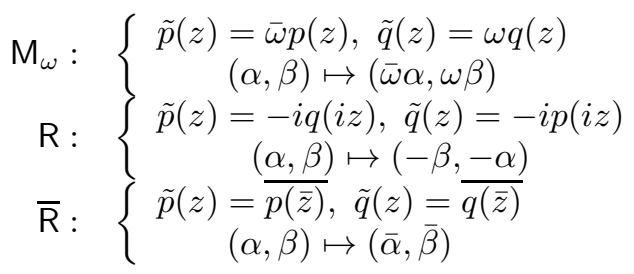

Nontrivial Bäcklund transformations were found by Kecker [4]

$$
\mathrm{B}_{\omega}:\left\{\begin{array}{c}
\tilde{p}(z)=p(z)-\bar{\omega} \frac{\omega \alpha-\bar{\omega} \beta+1}{\omega p(z)+\bar{\omega} q(z)-z} \\
\tilde{q}(z)=q(z)+\omega \frac{\omega \alpha-\bar{\omega} \beta+1}{\omega p(z)+\bar{\omega} q(z)-z} \\
(\alpha, \beta) \mapsto(\omega \beta-\bar{\omega}, \bar{\omega} \alpha+\omega)
\end{array}\right.
$$


(as long as the denominator does not vanish identically). By Bäcklund transformation we mean any repeated application of the above transformations. Bäcklund transformations act on pairs $(p, q)$, components $p$ and $q$, and also parameters $(\alpha, \beta)$. Under the Bäcklund transformation $\mathrm{B}_{\omega}$ the residues $\varrho=\operatorname{res}_{\lambda} p$ change as follows:

$$
\left.\begin{array}{rl}
\varrho \neq \bar{\omega}: & \operatorname{res}_{\lambda} \mathrm{B}_{\omega} p=\varrho \\
\varrho=\bar{\omega}: & \mathrm{B}_{\omega} p \text { is regular at } \lambda \text { and } \\
& \omega p+\bar{\omega} q-\left.z\right|_{z=\lambda}=0 \\
\omega p(\tilde{\lambda})+\bar{\omega} q(\tilde{\lambda})-\tilde{\lambda}=0 \\
(\tilde{\lambda} \text { not a pole of } p) \\
\omega p^{\prime}(\tilde{\lambda})+\bar{\omega} q^{\prime}(\tilde{\lambda})=-\omega \alpha+\bar{\omega} \beta
\end{array}\right\}: \begin{aligned}
& \operatorname{res}_{\tilde{\lambda}} \mathrm{B}_{\omega} p=\bar{\omega} \\
&
\end{aligned}
$$

\section{Rescaling}

3.1. Yosida functions. Let $a$ and $b>-1$ be real parameters. By definition, the class $\widetilde{\mathfrak{Y}}_{a, b}$ consists of all meromorphic functions $f$ such that the family $\left(f_{\kappa}\right)_{|\kappa|>1}$ of functions

$$
f_{\kappa}(\mathfrak{z})=\kappa^{-a} f\left(\kappa+\kappa^{-b} \mathfrak{z}\right)
$$

is normal on $\mathbb{C}$ in the sense of Montel, and all limit functions $\mathfrak{f}=\lim _{\kappa_{n} \rightarrow \infty} f_{\kappa_{n}}$ are $\not \equiv \infty$, at least one of them being non-constant. If, in addition, all limit functions are non-constant, then $f$ is said to belong to the Yosida class $\mathfrak{Y}_{a, b}$. The functions of class $\mathfrak{Y}_{0,0}$ were introduced by Yosida [12, and for arbitrary real parameters by the author [9]. The class $\mathfrak{Y}_{0,0}$ is universal in the sense that it contains all limit functions $\mathfrak{f}=\lim _{\kappa_{n} \rightarrow \infty} f_{\kappa_{n}}$ for $f \in \mathfrak{Y}_{a, b}$. The functions $f \in \widetilde{\mathfrak{Y}}_{a, b}$ have striking properties, for example they satisfy $f^{\sharp}(z)=O\left(|z|^{|a|+b}\right), T(r, f)=O\left(r^{2+2 b}\right)$, and $m(r, f)=O(\log r)$, and even $T(r, f) \asymp r^{2+2 b}$ and $m\left(r, 1 / f^{\prime}\right)=O(\log r)$ if $f \in \mathfrak{Y}_{a, b}$. For notations and results in Nevanlinna Theory the reader is referred to Hayman's monograph [3].

3.2. An application to the system (1). We quote from Shimomura's paper [], section 5.2, and also from section 6 in the author's paper 8 the following facts about the solutions to (4) and, obviously, also to the functions $w=p+q-z$ solving (3) and to $w=\bar{\varrho} p+\varrho q-z\left(\varrho^{3}=1\right)$ which solve similar equations:

- For $\delta>0$ sufficiently small, the discs $\triangle_{\delta}(\lambda)(\lambda \in \Lambda)$ are mutually disjoint; - $w=O(|z|)$ as $z \rightarrow \infty$ outside $\Lambda_{\delta}$ (for the definition of $\triangle_{\delta}$ and $\Lambda_{\delta}$ cf. ([6) ). It follows from (5) that the second condition is equivalent to

$$
|p|+|q|=O(|z|) \quad\left(z \rightarrow \infty, z \notin \Lambda_{\delta}\right)
$$

while (11) implies

$$
\left|p^{\prime}\right|+\left|q^{\prime}\right|=O\left(|z|^{2}\right) \quad\left(z \rightarrow \infty, z \notin \Lambda_{\delta}\right) .
$$

If $\lambda$ is any pole with residue $\operatorname{res}_{\lambda} p=\varrho$, then it follows from (7) that $f=\varrho p^{\prime}+p^{2}-\varrho z p$ is regular on $\triangle_{\delta}(\lambda)$, and $f(z)=O\left(|z|^{2}\right)$ on $\partial \triangle_{\delta}(\lambda)$ continues to hold on $\triangle_{\delta}(\lambda)$ by the Maximum Principle. This implies $\left|p^{\prime}\right|=O\left(|z|^{2}+|p|^{2}\right)$ on $\triangle_{\delta}(\lambda)$, and on combination with (16) this gives

$$
\frac{\left|p^{\prime}\right|}{|z|^{2}+|p|^{2}}+\frac{\left|q^{\prime}\right|}{|z|^{2}+|q|^{2}}=O(1) \quad(z \rightarrow \infty) \text { without restriction. }
$$

From (17) easily follows: 
THEOREM 1. The solutions $p$ and $q$ to equation (11) belong to the class $\widetilde{\mathfrak{Y}}_{1,1}$. The limit functions $\mathfrak{p}=\lim _{\kappa_{n} \rightarrow \infty} p_{\kappa_{n}}$ and $\mathfrak{q}=\lim _{\kappa_{n} \rightarrow \infty} q_{\kappa_{n}}$ solve

$$
\mathfrak{p}^{\prime}=-\mathfrak{q}^{2}-\mathfrak{p}, \mathfrak{q}^{\prime}=\mathfrak{p}^{2}+\mathfrak{q}, \quad \frac{1}{3}\left(\mathfrak{p}^{3}+\mathfrak{q}^{3}\right)+\mathfrak{p q}=\mathfrak{c}
$$

for some constant $\mathfrak{c}$ depending on $\left(\kappa_{n}\right)$.

Proof. Set $f(z)=p(z) / z, z=\kappa+\kappa^{-1} \mathfrak{z}$ and $f_{\kappa}(\mathfrak{z})=f(z)$. Then (17) implies

$f^{\sharp}(z) \leq \frac{\left|p^{\prime}(z)\right||z|^{-1}}{1+|p(z)|^{2}|z|^{-2}}+\frac{|p(z)||z|^{-2}}{1+|p(z)|^{2}|z|^{-2}}=|z| \frac{\left|p^{\prime}(z)\right|}{|z|^{2}+|p(z)|^{2}}+O(1)=O(1+|z|)$

and

$$
f_{\kappa}^{\sharp}(\mathfrak{z})=|\kappa|^{-1} f^{\sharp}\left(\kappa+\kappa^{-1} \mathfrak{z}\right)=O\left(1+|\kappa|^{-2}|\mathfrak{z}|\right)=O(|\mathfrak{z}|) \quad(\mathfrak{z} \rightarrow \infty) .
$$

By Marty's well-known criterion (see Ahlfors [1]), the family $\left(f_{\kappa}\right)_{|\kappa|>1}$ is normal on $\mathbb{C}$, and so is the family of functions $p_{\kappa}(\mathfrak{z})=\left(1+\kappa^{-2} \mathfrak{z}\right) f_{\kappa}(\mathfrak{z})$. The same is true for the family $\left(q_{\kappa}\right)$. The limit functions are finite since $\mathfrak{p}$ and $\mathfrak{q}$ have simple poles at $\mathfrak{z}=0$ with residues $\varrho$ and $-\bar{\varrho}$, respectively, if $\lim _{\kappa_{n} \rightarrow \infty}\left|\kappa_{n}\right| \operatorname{dist}\left(\kappa_{n}, \Lambda\right)=0$, while $\mathfrak{p}(0)$ and $\mathfrak{q}(0)$ are finite if $\liminf _{\kappa_{n} \rightarrow \infty}\left|\kappa_{n}\right| \operatorname{dist}\left(\kappa_{n}, \Lambda\right)>0$. Obviously, $\mathfrak{p}$ and $\mathfrak{q}$ solve (18) with

$$
\mathfrak{c}=\lim _{n \rightarrow \infty} \kappa_{n}^{-3} H\left(\kappa_{n}\right) \quad \text { if } \liminf _{\kappa_{n} \rightarrow \infty}\left|\kappa_{n}\right| \operatorname{dist}\left(\kappa_{n}, \Lambda\right)>0
$$

and

$$
\mathfrak{c}=\lim _{n \rightarrow \infty} \lambda_{n}^{-3}\left(2 \mathbf{h}\left(\lambda_{n}\right)+\frac{1}{3} \lambda_{n}^{3}\right) \quad \text { if } \lim _{\kappa_{n} \rightarrow \infty}\left|\kappa_{n}\right|\left|\kappa_{n}-\lambda_{n}\right|=0
$$

for some sequence of poles $\left(\lambda_{n}\right)$.

Remark. Constant limit functions are $(0,0)$ and $(-\omega,-\bar{\omega})$ with $\omega^{3}=1$. They correspond to $\mathfrak{c}=0$ and $\mathfrak{c}=\frac{1}{3}$, respectively.

3.3. The cluster set. By definition, the cluster set $\mathfrak{C}(p, q) \subset \mathbb{C}$ of any non-trivial solution $(p, q)$ to (1) consists of all limits (19).

ThEOREM 2. The cluster set $\mathfrak{C}(p, q)$ is closed, bounded, and connected, and contains all limits (20).

Proof. For $\delta>0$ sufficiently small, the closed discs $\bar{\triangle}_{\delta}(\lambda)$ about the poles $\lambda \neq 0$ are mutually disjoint, hence any two points $a, b \in D_{\delta}=\mathbb{C} \backslash \bigcup_{\lambda \in \Lambda} \bar{\triangle}_{\delta}(\lambda)$ may be joined by a curve that is contained in $D_{\delta} \cap\{z:|z| \geq \min \{|a|,|b|\}\}$. We denote the corresponding cluster set of $z^{-3} H(z)$ as $z \rightarrow \infty$ on $D_{\delta}$ by $\mathfrak{C}_{\delta}(p, q)$, and note that $\mathfrak{C}(p, q)=\bigcup_{\delta>0} \mathfrak{C}_{\delta}(p, q)$. The cluster sets $\mathfrak{C}_{\delta}(p, q)$ are closed, bounded in $\mathbb{C}$ since $z^{-3} H(z)$ is uniformly bounded on $D_{\delta}$, and connected by the special property of $D_{\delta}$. Since $\mathfrak{C}_{\eta}(p, q) \supset \mathfrak{C}_{\delta}(p, q)$ for $0<\eta<\delta$, it remains to show that

$$
\mathfrak{C}_{\eta}(p, q) \subset \mathfrak{C}_{\delta}(p, q) \quad(0<\eta<\delta \text { sufficiently small }),
$$

and that the limits (20) belong to $\mathfrak{C}_{\delta}(p, q)$. If

$$
\eta \leq\left|\kappa_{n}\right| \operatorname{dist}\left(\kappa_{n}, \Lambda\right)=\left|\kappa_{n}\right|\left|\kappa_{n}-\lambda_{n}\right|<\delta
$$

holds for some $\lambda_{n} \in \Lambda$, and if $\mathfrak{p}=\lim _{\kappa_{n} \rightarrow \infty} p_{\kappa_{n}}, \mathfrak{q}=\lim _{\kappa_{n} \rightarrow \infty} q_{\kappa_{n}}$ and $\mathfrak{c}=\lim _{\kappa_{n} \rightarrow \infty} \kappa_{n}^{-3} H\left(\kappa_{n}\right)$ exist, we replace $\kappa_{n}$ by $\lambda_{n}$ with the following effect: from $\lambda_{n}=\kappa_{n}+\kappa_{n}^{-1} \mathfrak{z}_{n}$ with $\left(\mathfrak{z}_{n}\right)$ bounded, hence $\mathfrak{z}_{n} \rightarrow \mathfrak{z}_{0}$ as we may assume, it follows that $\lambda_{n}^{-1}=(1+o(1)) \kappa_{n}^{-1}$, $p_{\kappa_{n}}(\mathfrak{z})=(1+o(1)) p_{\lambda_{n}}\left(\mathfrak{z}-\mathfrak{z}_{0}+o(1)\right)$ and $q_{\kappa_{n}}(\mathfrak{z})=(1+o(1)) q_{\lambda_{n}}\left(\mathfrak{z}-\mathfrak{z}_{0}+o(1)\right)$, hence

$$
\hat{\mathfrak{p}}(\mathfrak{z})=\lim _{\lambda_{n} \rightarrow \infty} p_{\lambda_{n}}(\mathfrak{z})=\mathfrak{p}\left(\mathfrak{z}+\mathfrak{z}_{0}\right), \hat{\mathfrak{q}}(\mathfrak{z})=\lim _{\lambda_{n} \rightarrow \infty} q_{\lambda_{n}}(\mathfrak{z})=\mathfrak{q}\left(\mathfrak{z}+\mathfrak{z}_{0}\right)
$$


and

$$
\frac{1}{3}\left(\hat{\mathfrak{p}}^{3}+\hat{\mathfrak{q}}^{3}\right)+\hat{\mathfrak{p}} \hat{\mathfrak{q}}=\mathfrak{c}=\lim _{n \rightarrow \infty} \lambda_{n}^{-3}\left(2 \mathbf{h}\left(\lambda_{n}\right)+\frac{1}{3} \lambda_{n}^{3}\right) .
$$

Finally, if we start with sequences $\left(p_{\lambda_{n}}\right)$ and $\left(q_{\lambda_{n}}\right)$ with $\lambda_{n} \in \Lambda$, we may as well consider $\left(p_{\kappa_{n}}\right)$ and $\left(q_{\kappa_{n}}\right)$ with $\left|\kappa_{n}\right|\left|\kappa_{n}-\lambda_{n}\right|=\delta$, hence

$$
\mathfrak{c}=\lim _{\lambda_{n} \rightarrow \infty} \lambda_{n}^{-3}\left(2 \mathbf{h}\left(\lambda_{n}\right)+\frac{1}{3} \lambda_{n}^{3}\right)=\lim _{\kappa_{n} \rightarrow \infty} \kappa_{n}^{-3} H\left(\kappa_{n}\right) \in \mathfrak{C}_{\delta}(p, q) .
$$

Combining both arguments this also proves (21).

3.4. An algebraic curve. The algebraic curve

$$
\frac{1}{3}\left(u^{3}+v^{3}\right)+u v=c
$$

- is reducible if $c=\frac{1}{3}: u^{3}+v^{3}+3 u v-1=\prod_{\varrho^{3}=1}(v+\varrho u-\varrho)$,

- has genus zero if $c=0$, and

- has genus one otherwise.

In the first case $\left(c=\frac{1}{3}\right)$ the corresponding Hamiltonian system

$$
u^{\prime}=-v^{2}-u, v^{\prime}=u^{2}+v
$$

has solutions given by $\frac{u+\omega}{u+\bar{\omega}}=e^{i \sqrt{3} t}\left(\omega=\frac{1}{2}(-1+i \sqrt{3})\right)$ and $v=1-u$, and similar expressions if $v=\bar{\varrho}-\varrho u$ and $v=\varrho-\bar{\varrho} u$, respectively. It is just important to know that the poles form a $2 \pi / \sqrt{3}$-periodic sequence with fixed residue. If the genus is zero $(c=0)$, the system (23) is solved by $u=\frac{-3 \omega e^{2 t}}{e^{3 t}+1}$ and $v=\frac{-3 \bar{\omega} e^{t}}{e^{3 t}+1}$, with poles forming a $2 \pi i / 3$-periodic sequence, this time with alternating residues. In the remaining cases, the solutions to (23) are elliptic functions parametrising the curve (22); they have elliptic order three with corresponding lattice $L_{c}$ depending only on $c$.

\section{VALUe Distribution}

4.1. The order of growth. We note as a corollary to Theorem 1 the following estimate:

THEOREM 3. The solutions to equation (1) have order of growth at most four:

$$
T(r, p)+T(r, q)=O\left(r^{4}\right) .
$$

Proof. From $f^{\sharp}(z)=O(|z|)$ and $T(r, p)=T(r, f)+O(\log r)$ for $f(z)=p(z) / z$ we easily obtain (24) when using the Ahlfors-Shimizu form of the Nevanlinna characteristic,

$$
T(r, f)=\int_{0}^{r} A(t) \frac{d t}{t} \quad \text { with } \quad A(t)=\frac{1}{\pi} \int_{|z|<t} f^{\sharp}(z)^{2} d x d y .
$$

REMARK. This result corresponds to the meanwhile well-established estimate 6, 8 for the order of growth of the fourth Painlevé transcendents. 
4.2. Solutions of maximal growth. Suppose that the cluster set $\mathfrak{C}(p, q)$ contains some parameter $\lim _{\kappa_{n} \rightarrow \infty} H\left(\kappa_{n}\right) \kappa_{n}^{-3} \neq 0, \frac{1}{3}$. Since $H(z) z^{-3}$ is varying slowly, this following from

$$
\frac{d}{d z} H(z) z^{-3}=-3 H(z) z^{-4}+p(z) q(z) z^{-3}=O\left(|z|^{-1}\right) \quad \text { outside } \Lambda_{\delta},
$$

given $\epsilon>0$ sufficiently small there exists $\eta>0$ such that the re-scaling procedure for $\left|\tilde{\kappa}_{n}-\kappa_{n}\right|<\eta\left|\kappa_{n}\right|$ leads to limit functions $\mathfrak{p}$ and $\mathfrak{q}$ with

$$
\mathfrak{p}^{3}+\mathfrak{q}^{3}+3 \mathfrak{p q}=3 \mathfrak{c} \quad\left(\min \left\{|\mathfrak{c}|,\left|\mathfrak{c}-\frac{1}{3}\right|\right\} \geq \epsilon,|\mathfrak{c}| \leq 1 / \epsilon\right) .
$$

Now $\mathfrak{p}$ and $\mathfrak{q}$ are elliptic functions with fundamental parallelogram $P_{\mathfrak{c}}$, whose diameter and area is uniformly bounded and bounded away from zero. It is thus easily deduced that the disc $\left|z-\kappa_{n}\right|<\eta\left|\kappa_{n}\right|$ contains at least const $\left|\kappa_{n}\right|^{4}$ poles of $p$ (it is almost the same to say that the disc $|z|<r$ contains $\sim \pi r^{2}$ lattice points $m+i n$ ), hence

$$
n\left(2\left|\kappa_{n}\right|, p\right) \geq \text { const }\left|\kappa_{n}\right|^{4}
$$

holds. On combination with $n(r, \Lambda)=O\left(r^{4}\right)$ we thus have:

THEOREm 4. Let $(p, q)$ be any solution to equation (1). Then $\mathfrak{C}(p, q) \not \subset\left\{0, \frac{1}{3}\right\}$ implies $n\left(r_{k}, \Lambda\right) \asymp r_{k}^{4}$, at least on some sequence $r_{k} \rightarrow \infty$.

4.3. The distribution of residues. Leaving the rational solutions aside we henceforth will consider only transcendental solutions. From

$$
q^{2}=-p^{\prime}-z p-\alpha=-p\left(z+p^{\prime} / p\right)-\alpha
$$

it follows by the usual rules of Nevanlinna Theory that $2 m(r, q) \leq m(r, p)+O(\log r)$, and in the same manner $2 m(r, p) \leq m(r, q)+O(\log r)$ is obtained, hence $m(r, p)+$ $m(r, q)=O(\log r), T(r, p)=N(r, \Lambda)+O(\log r)$, and $T(r, q)=N(r, \Lambda)+O(\log r)$ hold; here $N(r, \Lambda)$ denotes the common Nevanlinna counting function of poles. Cauchy's Residue Theorem yields

$$
\frac{1}{2 \pi i} \int_{C_{r}} p(z) d z=n\left(r, \Lambda^{1}\right)+\varrho n\left(r, \Lambda^{\varrho}\right)+\bar{\varrho} n\left(r, \Lambda^{\bar{\varrho}}\right) \quad\left(\varrho=\frac{1}{2}(-1+i \sqrt{3})\right),
$$

provided the circle $C_{r}:|z|=r$ intersects no pole. If $C_{r}$ intersects the disc $\triangle_{\delta}(\lambda)$, we replace the arc $C_{r} \cap \triangle_{\delta}(\lambda)$ by the sub-arc of $\partial \triangle_{\delta}(\lambda)$ outside $C_{r}$ if $|\lambda| \leq r$, and inside $C_{r}$ otherwise. This way we obtain a simple closed curve $\Gamma_{r}$ such that

$$
|p(z)|+|q(z)|=O(|z|) \text { holds on } \Gamma_{r},
$$

without changing the integral (25). The length of the part of $\Gamma_{r}$ restricted to any sector of central angle $\Theta$ is $O(r \Theta)$, hence the integral is $O\left(r^{2}\right)$. Taking real and imaginary parts, we obtain

$$
\begin{aligned}
n\left(r, \Lambda^{1}\right)-\frac{1}{2}\left(n\left(r, \Lambda^{\varrho}\right)+n\left(r, \Lambda^{\bar{\varrho}}\right)\right) & =O\left(r^{2}\right) \\
\frac{\sqrt{3}}{2}\left(n\left(r, \Lambda^{\varrho}\right)-n\left(r, \Lambda^{\bar{\varrho}}\right)\right) & =O\left(r^{2}\right) \quad\left(\varrho=\frac{1}{2}(-1+i \sqrt{3})\right) .
\end{aligned}
$$

TheOrem 5. For any transcendental solution $(p, q)$ to equation (1), the counting functions $n\left(r, \Lambda^{\varrho}\right)$ are equal up to a term $O\left(r^{2}\right)$, that is, we have

$$
n\left(r, \Lambda^{\varrho}\right)=\frac{1}{3} n(r, \Lambda)+O\left(r^{2}\right) \quad\left(\varrho^{3}=1\right) .
$$

In particular, if one kind of poles is missing, $p$ and $q$ have order of growth at most two: $T(r, p)+T(r, q)=O\left(r^{2}\right)$. 
4.4. Strings of poles. Re-scaling along any sequence of poles with corresponding limit $\mathfrak{c} \in\left\{0, \frac{1}{3}\right\}$ leads to the following situation: given $\epsilon>0$ and $R>0$ there exists $r_{0}>0$, such that for any pole $\lambda_{0}$ in $|z|>r_{0}$, the disc $\triangle_{R}(\lambda)$ contains the poles

$$
\lambda_{k}=\lambda_{0}+k\left(\varpi+\epsilon_{k}\right) \lambda_{0}^{-1} \quad\left(\left|\epsilon_{k}\right|<\epsilon,-k_{1} \leq k \leq k_{2}\right)
$$

with $\varpi=2 \pi i / 3$ and $\varpi=2 \pi / \sqrt{3}$, respectively, and no others $\left(k_{1}=k_{2}=[R /|\varpi|]\right.$ if $R$ is large and not an integer multiple of $|\varpi|)$. In other words, for $\mathfrak{C}(p, q) \subset\left\{0, \frac{1}{3}\right\}$, each pole $\lambda$ with $|\lambda|$ sufficiently large belongs to some unique sequence $\left(\lambda_{n}\right)$, called string of poles; it satisfies the approximative recursion

$$
\lambda_{n+1}=\lambda_{n} \pm(\varpi+o(1)) \lambda_{n}^{-1} .
$$

Setting $\sigma_{n}=\lambda_{n}^{2}$ we obtain $\sigma_{n+1}=\sigma_{n} \pm 2 \varpi+o(1), \sigma_{n}=2 n \varpi+o(n)$,

$$
\lambda_{n}=\sqrt{2 n|\varpi|}(1+o(1)) \quad \text { and } \quad \arg \lambda_{n}=\frac{1}{2} \arg \varpi+o(1) \bmod \frac{\pi}{2} ;
$$

the counting function of $\left\{\lambda_{n}\right\}$ is given by $n\left(r,\left\{\lambda_{n}\right\}\right) \sim \frac{r^{2}}{2|\varpi|}$. We note that the estimate $n(r, \Lambda)=O\left(r^{4}\right)$ now can be completed:

Theorem 6. Any transcendental solution $(p, q)$ to (1) satisfies

$$
r^{2}=O(n(r, \Lambda)) \text { and } n(r, \Lambda)=O\left(r^{4}\right) .
$$

Proof. This is obvious if $\Lambda$ contains a full string. In any case the re-scaling method shows that to any pole $\lambda$ of sufficiently large modulus there exists some pole $\tilde{\lambda}$ satisfying $|\lambda|<|\tilde{\lambda}|<|\lambda|+O\left(|\lambda|^{-1}\right)$, this implying $r^{2}=O(n(r, \Lambda))$.

\section{Asymptotic EXPANsions AND POLE-FreE SECTORS}

5.1. Pole-free sectors. Let $(p, q)$ be any transcendental solution to (1). If $p$ has no poles on some sector $S:|\arg z-\hat{\theta}|<\theta,|z|>r_{0}$, the re-scaling procedure with $\kappa_{n} \in S_{\delta}:|\arg z-\hat{\theta}|<\theta-\delta$ leads to solutions $(\mathfrak{p}, \mathfrak{q})$ without poles, hence to constant solutions. The possible constants are $(0,0)$ and $(-\tau,-\bar{\tau})$, again with $\tau^{3}=1$, hence we have either $p(z)=o(|z|)$ and $q(z)=o(|z|)$, or else $p(z)=-\tau z+o(|z|)$ and $q(z)=-\bar{\tau} z+o(|z|)$ as $z \rightarrow \infty$ on $S$. In each case we will prove that this leads to asymptotic expansions on certain pole-free sectors.

5.2. Asymptotics on pole-free sectors with $\mathfrak{C}(\mathbf{p}, \mathbf{q})=\{\mathbf{0}\}$. In the first case the following holds in more generality:

TheOREM 7. Suppose that $p(z)=o(|z|)$ and $q(z)=o(|z|)$ hold as $z \rightarrow \infty$ on some ray $\hat{\sigma}: \arg z=\hat{\theta} \not \equiv \frac{\pi}{4} \bmod \frac{\pi}{2}$. Then $p, q$, and $H$ have asymptotic expansions

$$
\begin{aligned}
p(z) & \sim-\frac{\alpha}{z}-\frac{\alpha+\beta^{2}}{z^{3}}-\frac{3 \alpha+\beta^{2}+2 \alpha^{2} \beta}{z^{5}}+\cdots \\
q(z) & \sim-\frac{\beta}{z}+\frac{\beta-\alpha^{2}}{z^{3}}+\frac{3 \beta-\alpha^{2}-2 \alpha \beta^{2}}{z^{5}}+\cdots \\
H(z) & \sim-\frac{\alpha \beta}{z}-\frac{\alpha^{3}+\beta^{3}}{3 z^{3}}+\cdots \quad(z \rightarrow \infty)
\end{aligned}
$$

on the sector $\Sigma_{\nu}:\left|\arg z-\nu \frac{\pi}{2}\right|<\frac{\pi}{4}$ that contains the ray $\hat{\sigma}$.

Proof. From our hypothesis it also follows that $H(z)=o\left(|z|^{3}\right)$ holds along $\hat{\sigma}$. Rescaling along any sequence $\left(\kappa_{n}\right)$ on $\hat{\sigma}$ yields limit functions $\mathfrak{p}$ and $\mathfrak{q}$ satisfying

$$
\mathfrak{p}^{\prime}=-\mathfrak{q}^{2}-\mathfrak{p}, \mathfrak{q}^{\prime}=\mathfrak{p}^{2}+\mathfrak{q}, \mathfrak{p}(0)=\mathfrak{q}(0)=0, \text { and } \mathfrak{p}^{3}+\mathfrak{q}^{3}+3 \mathfrak{p} \mathfrak{q}=0
$$


hence $\mathfrak{p}=\mathfrak{q} \equiv 0$. Thus there are pole-free discs $\left|z-r e^{i \hat{\theta}}\right|<r^{-1} \kappa(r)$ such that $\kappa(r) \rightarrow \infty$ as $r \rightarrow \infty$. To identify the maximal pole-free sector that contains $\hat{\sigma}$ (if any), we start with $r_{0}>0$ sufficiently large and define the sequence $\left(r_{n}\right)$ inductively by $r_{n+1}=r_{n}+4 r_{n}^{-1}$. By $\theta_{n}$ we denote the largest number such that

$$
A_{n}=\left\{z: r_{n} \leq|z| \leq r_{n+1}, \hat{\theta} \leq \arg z<\theta_{n}\right\}
$$

contains no pole of $p$, noting that $r_{n} \theta_{n} \sim \kappa\left(r_{n}\right) \rightarrow \infty$. We may assume that there exists some pole $\tilde{\lambda}_{0}$ on $\partial A_{0}$, hence $\arg \tilde{\lambda}_{0}=\theta_{0}$. The same is true at least for some sub-sequence $\partial A_{n_{k}}$. Re-scaling along the sequence $\left(\lambda_{n_{k}}\right)$ then yields limit functions $\tilde{\mathfrak{p}}$ and $\tilde{\mathfrak{q}}$ that have simple poles at $\mathfrak{z}=0$ and satisfy $\tilde{\mathfrak{p}}^{3}+\tilde{\mathfrak{q}}^{3}+3 \tilde{\mathfrak{p}} \tilde{\mathfrak{q}}=3 \mathfrak{c}$. Since $A_{n_{k}}$ is large with respect to the metric $d s=|z||d z|$ and contains no pole, $\tilde{\mathfrak{p}}$ and $\tilde{\mathfrak{q}}$ cannot be elliptic functions, and this and $\lim _{z \rightarrow \infty} H(z) z^{-3}=0$ on $\bigcup_{n} A_{n}$ implies $\mathfrak{c}=0$, hence the algebraic curve (22) has genus zero. It follows from Hurwitz' Theorem on the poles of limit functions that to each pole $\tilde{\lambda}_{n_{k}}$ there exist five poles

$$
z_{k, \nu}=\tilde{\lambda}_{n_{k}}+\nu(\varpi+o(1)) \tilde{\lambda}_{n_{k}}^{-1} \quad(-2 \leq \nu \leq 2, \varpi= \pm 2 \pi i / 3)
$$

on $\left|z-\tilde{\lambda}_{n}\right|<5\left|\tilde{\lambda}_{n}\right|^{-1}$, and no others. Since $z_{k, \pm 2}$ does not belong to the annulus $r_{n_{k}} \leq|z| \leq r_{n_{k}+1}$, it follows that $A_{n_{k}-1}$ and $A_{n_{k}+1}$, hence each $A_{n}$ contains some pole $\tilde{\lambda}_{n}$ on its boundary, and $\left(\tilde{\lambda}_{n}\right)$ is a sub-sequence of some sequence $\left(\lambda_{n}\right)$ of poles satisfying the approximate recursion (27). Thus $\arg \lambda_{n}$ and $\theta_{n}$ approach $(2 \nu+1) \frac{\pi}{4}>\hat{\theta}$ for some $\nu$. The same argument applies to the regions $\left\{z: r_{n} \leq|z| \leq\right.$ $\left.r_{n+1}, \theta_{n}<\arg z \leq \hat{\theta}\right\}$, this showing that the sectors $\Sigma_{\nu}$ are the natural pole-free sectors in the sense that for every $\delta>0, S_{\delta}=\left\{z:\left|\arg z-\nu \frac{\pi}{2}\right|<\frac{\pi}{4}-\delta\right\}$ contains only finitely many poles.

For $z \in S_{\delta}$ sufficiently large we set $\max \{|p(z)|,|q(z)|\}=\epsilon|z|$. Since

$$
|p(z)|+|q(z)|=o(|z|) \quad\left(z \rightarrow \infty \text { on } S_{\frac{\delta}{2}}\right),
$$

we may assume $\epsilon=\epsilon(z)<\frac{1}{2}$. From (11) and $\left|p^{\prime}(z)\right|+\left|q^{\prime}(z)\right| \rightarrow 0$ as $z \rightarrow \infty$ on $S_{\delta}$ (this following from (29) and Cauchy's Theorem), hence $\left|p^{\prime}(z)\right|+\left|q^{\prime}(z)\right|<1$, say, we obtain

$$
|z p(z)|<\epsilon^{2}|z|^{2}+|\alpha|+1 \text { and }|z q(z)|<\epsilon^{2}|z|^{2}+|\beta|+1 .
$$

This yields $\left(\epsilon-\epsilon^{2}\right)|z|^{2}<K$ and $\epsilon<2 K|z|^{-2}$, hence

$$
|p(z)|+|q(z)|=O\left(|z|^{-1}\right) \quad \text { and } \quad\left|p^{\prime}(z)\right|+\left|q^{\prime}(z)\right|=O\left(|z|^{-2}\right) \quad\left(z \rightarrow \infty \text { on } S_{\delta}\right),
$$

again by Cauchy's Theorem. From (11) it then follows that

$$
z p(z)+\alpha=O\left(|z|^{-2}\right) \text { and } z q(z)+\beta=O\left(|z|^{-2}\right)
$$

as $z \rightarrow \infty$ on $S_{\delta}$. Now assume that

$$
\begin{aligned}
& p(z)=-\alpha z^{-1}+\sum_{\nu=1}^{n} a_{\nu} z^{-2 \nu-1}+O\left(|z|^{-2 n-3}\right)=\phi_{n}(z)+O\left(|z|^{-2 n-3}\right) \\
& q(z)=-\beta z^{-1}+\sum_{\nu=1}^{n} b_{\nu} z^{-2 \nu-1}+O\left(|z|^{-2 n-3}\right)=\psi_{n}(z)+O\left(|z|^{-2 n-3}\right)
\end{aligned}
$$

has already been proved. From (1) it then follows that

$$
\begin{gathered}
z p(z)+\alpha=-q(z)^{2}-p^{\prime}(z)=-\psi_{n}(z)^{2}-\phi_{n}^{\prime}(z)+O\left(|z|^{-2 n-4}\right) \\
z q(z)+\beta=-p(z)^{2}+q^{\prime}(z)=-\phi_{n}(z)^{2}+\psi_{n}^{\prime}(z)+O\left(|z|^{-2 n-4}\right)
\end{gathered}
$$


holds. Since $\phi_{n}^{\prime}, \psi_{n}^{\prime}, \phi_{n}^{2}$, and $\psi_{n}^{2}$ are even functions, (30) holds with $n$ replaced by $n+1$. A more detailed computation(11) then gives (28).

5.3. Asymptotics on pole-free sectors with $\mathfrak{C}(\mathbf{p}, \mathbf{q})=\left\{\frac{1}{3}\right\}$. The other cases are yet easier to deal with, since the principal terms $-\tau z$ and $-\bar{\tau} z\left(\tau^{3}=1\right)$ are already known. Details are left to the reader. There is, however, one remarkable difference: the natural pole-free sectors now are $\Sigma_{\nu}: \nu \frac{\pi}{2}<\arg z<(\nu+1) \frac{\pi}{2}$, and these sectors are bordered by sequences of poles $\lambda_{n}$ satisfying (27) with $\varpi=\frac{2 \pi}{\sqrt{3}}$ and having counting function $n\left(r,\left\{\lambda_{n}\right\}\right) \sim \frac{\sqrt{3} r^{2}}{4 \pi}$. We also note that in the present case the algebraic curve (22) is reducible $\left(c=\frac{1}{3}\right)$.

TheOrem 8. Suppose that $p(z)=-\tau z+o(|z|)$ and $q(z)=-\bar{\tau} z+o(|z|)\left(\tau^{3}=1\right)$ hold as $z \rightarrow \infty$ on some ray $\hat{\sigma}: \arg z=\hat{\theta} \not \equiv 0 \bmod \frac{\pi}{2}$. Then $p, q$, and $H$ have asymptotic expansions

$$
\begin{aligned}
p(z) & \sim-\tau z+\frac{\alpha+2 \bar{\tau} \beta+\tau}{3 z}+\frac{3 \alpha+2 \bar{\tau} \beta+2 \bar{\tau} \alpha^{2}+3 \beta^{2}+4 \tau \alpha \beta-2 \tau}{9 g^{3}}+\cdots \\
q(z) & \sim-\bar{\tau} z+\frac{\beta+2 \tau \alpha-\bar{\tau}}{3 z}-\frac{3 \beta+2 \tau \alpha-2 \tau \beta^{2}-3 \alpha^{2}-4 \bar{\tau} \alpha \beta+2 \bar{\tau}}{9 z^{3}}+\cdots \\
H(z) & \sim \frac{z^{3}}{3}-(\tau \alpha+\bar{\tau} \beta) z+\frac{\tau \alpha^{2}+\bar{\tau} \beta^{2}+\alpha \beta-1}{3 z}+\cdots
\end{aligned}
$$

as $z \rightarrow \infty$ on the sector $\Sigma_{\nu}: \nu \frac{\pi}{2}<\arg z<(\nu+1) \frac{\pi}{2}$ that contains $\hat{\sigma}$.

5.4. Asymptotics on adjacent pole-free sectors. Both theorems may be completed as follows:

TheOREM 9. Suppose that the same asymptotics (28) and (31) hold on adjacent sectors $\Sigma_{\nu}$ and $\Sigma_{\nu+1}$, respectively. Then this is true on $\left(\overline{\Sigma_{\nu} \cup \Sigma_{\nu+1}}\right)^{\circ}$.

Proof. In both cases, $g(z)=\exp \int H(z) d z$ is an entire function of finite order $(\leq 4)$ having simple zeros at the poles of $p$ and $q$. Taking into account the asymptotics (28) and (31) of $H$, we consider

$$
f(z)=z^{\alpha \beta} g(z) \quad \text { and } \quad f(z)=g(z) z^{-\left(\tau \alpha^{2}+\bar{\tau} \beta^{2}+\alpha \beta-1\right) / 3} \exp \left(-\frac{z^{4}}{12}+\frac{1}{2}(\tau \alpha+\bar{\tau} \beta) z^{2}\right)
$$

in the respective cases. Then on $\left|\arg z-\bar{\theta}_{\nu}\right|<\frac{\pi}{8}$, say $\left(\arg z=\bar{\theta}_{\nu}\right.$ denotes the ray that separates $\Sigma_{\nu}$ and $\left.\Sigma_{\nu+1}\right), f$ has order of growth at most four,

$$
\limsup _{z \rightarrow \infty} \frac{\log \log |f(z)|}{\log |z|} \leq 4 \quad\left(\left|\arg z-\bar{\theta}_{\nu}\right|<\frac{\pi}{8}\right),
$$

and satisfies $\lim _{r \rightarrow \infty} f\left(r e^{i\left(\bar{\theta}_{\nu} \pm \delta\right)}\right)=C_{ \pm}$for $\delta=\frac{\pi}{10}$, say. The Phragmén-Lindelöf Principle(2) then shows that $C_{+}=C_{-}=C$, and $f(z)=C+o(1)$ holds as $z \rightarrow \infty$ on $\left|\arg z-\bar{\theta}_{\nu}\right|<\delta$. Hence $f$ and also $g$ has only finitely many zeros, and $p$ has only finitely many poles on that sector, that is, $p$ and $q$ have an asymptotic expansion on $|\arg z-\hat{\theta}|<\delta$ which coincides with those on the sectors $\Sigma_{\nu}$ and $\Sigma_{\nu+1}$.

\footnotetext{
${ }^{1}$ Many of the computations at various places were performed with the help of maple.

${ }^{2}$ The version we will use can easily be derived from the following standard version: Let $f$ be holomorphic on the domain $D:|z|>r_{0}$, $\operatorname{Im} z>0$, with continuous boundary values on $\left(-\infty,-r_{0}\right) \cup\left(r_{0},+\infty\right)$. If $f$ satisfies $\limsup _{z \rightarrow \infty} \frac{\log |f(z)|}{|z|}=0$ on $D, \lim _{x \rightarrow+\infty} f(x)=a$ and $\lim _{x \rightarrow-\infty} f(x)=b$, then $a=b$ and $\lim _{z \rightarrow \infty} f(z)=a$ holds on $D$.
} 
5.5. Bäcklund transformations and asymptotics. Under the Bäcklund transformation $\mathrm{B}_{\omega}$ the asymptotics (31) changes as follows:

$$
p(z) \sim-\tau z \text { and }\left\{\begin{array} { l } 
{ \omega = \overline { \tau } } \\
{ \omega \neq \overline { \tau } }
\end{array} \text { implies } \mathrm { B } _ { \omega } p ( z ) \sim \left\{\begin{array}{l}
-\tau z \\
-\bar{\tau} z .
\end{array}\right.\right.
$$

5.6. Existence and uniqueness of asymptotic expansions. In Theorem 7 and 8 it was shown that specific solutions have asymptotic expansions on certain sectors of central angle $\frac{\pi}{2}$. This should not be confused with our next result about existence and uniqueness of solutions having asymptotic expansions.

TheOrem 10. To any half-plane $\left|\arg z-(2 \nu+1) \frac{\pi}{4}\right|<\frac{\pi}{2}$ and $\left|\arg z-\nu \frac{\pi}{2}\right|<\frac{\pi}{2}$ there exists a unique solution to (1) with prescribed asymptotics (28) and (31), respectively.

Proof. To prove existence we set, in the first case (28), $t=z^{2}, p(z)=t^{-\frac{1}{2}} u(t)^{2}$, and $q(z)=t^{-\frac{1}{2}} v(t)^{2}$ to obtain

$$
\dot{u}=-\frac{\alpha+u^{2}}{4 u}+\frac{u^{2}-v^{4}}{4 t u}, \dot{v}=\frac{\beta+v^{2}}{4 v}+\frac{u^{4}+v^{2}}{4 t v} .
$$

Then given any half plane $H$, Theorem 14.1 in Wasow's monograph 11 applies to the corresponding system for $x=u-\sqrt{-\alpha}$ and $y=v-\sqrt{-\beta}$ in $H$; it yields existence of some solution having asymptotic expansion $u \sim \sqrt{-\alpha}+\sum_{k=1}^{\infty} a_{k} t^{-k}$, $v \sim \sqrt{-\beta}+\sum_{k=1}^{\infty} b_{k} t^{-k}$ as $t \rightarrow \infty$ on $H$. Thus (10) has some solution with asymptotic expansion (necessarily given by (28)) on $S=\sqrt{H}$. If, however, $S$ contains some ray $\hat{\sigma}_{\nu}: \arg z=\nu \frac{\pi}{2}$, then by Theorem 7 , the asymptotic expansion extends to the half-plane $\left|\arg z-\nu \frac{\pi}{2}\right|<\frac{\pi}{2}$. We note, however, that the proof only works if $\alpha \beta \neq 0$. In the trivial case $\alpha=\beta=0$ we have $p \equiv q \equiv 0$. In case of $\alpha=0$ and $\beta \neq 0$, say, we set $t=z^{2}, p(z)=t^{-3 / 2} u(t)^{2}$, and $q(z)=t^{-1 / 2} v(t)^{2}$ to obtain the system

$$
\dot{u}=-\frac{u^{2}+v^{4}}{4 u}+\frac{3 u}{4 t}, \dot{v}=\frac{v^{2}+\beta}{4 v}+\frac{v}{4 t}+\frac{u}{4 t^{3} v} .
$$

Again, Theorem 14.1 in [11] applies to the system for $x=u-i \beta, y=v-\sqrt{-\beta}$ $\left(u^{2}+v^{4}=v^{2}+\beta=0\right.$ is solved by $\left.v=\sqrt{-\beta}, u= \pm i \beta\right)$.

In the same way one may deal with the second case (31): just set $t=z^{2}, p(z)=$ $t^{\frac{1}{2}} u(t)^{2}$, and $q(z)=t^{\frac{1}{2}} v(t)^{2}$ to obtain

$$
\dot{u}=-\frac{u^{2}+v^{4}}{4 u}-\frac{\alpha+u^{2}}{4 t u}, \dot{v}=\frac{u^{4}+v^{2}}{4 v}+\frac{\beta-v^{2}}{4 t v},
$$

and apply Theorem 14.1 in [11] to the system for $x=u-\sqrt{-\tau}, y=v-\sqrt{-\bar{\tau}}$ (the non-trivial solutions to $\xi+\eta^{2}=\xi^{2}+\eta=0$ are $(\xi, \eta)=(-\tau,-\bar{\tau})$ with $\left.\tau^{3}=1\right)$.

To prove uniqueness we consider two solutions $\left(p_{1}, q_{1}\right)$ and $\left(p_{2}, q_{2}\right)$ having the same asymptotic expansion on some sector $S$. Then $u=p_{1}-p_{2}$ and $v=q_{1}-q_{2}$ tend to zero faster than every power $z^{-n}$, and $\mathfrak{u}=\left(\begin{array}{l}u \\ v\end{array}\right)$ solves $z^{-1} \mathfrak{u}^{\prime}=A(z) \mathfrak{u}$ with $A(\infty)=\left(\begin{array}{cc}-1 & 0 \\ 0 & 1\end{array}\right)$ (eigenvalues \pm 1 ) and $A(\infty)=\left(\begin{array}{cc}-1 & -2 \bar{\tau} \\ -2 \tau & 1\end{array}\right)$ (eigenvalues $\pm \sqrt{5})$, respectively. In every sector $\left|\arg z-\nu \frac{\pi}{2}\right|<\frac{\pi}{4}$ and $\left|\arg z-(2 \nu+1) \frac{\pi}{4}\right|<\frac{\pi}{4}$, respectively, systems of this kind have some solution $\mathfrak{u}_{1}$ that tends to infinity, 
and also some non-trivial solution $\mathfrak{u}_{2}$ (called sub-dominant) that tends to zero exponentially as $z \rightarrow \infty$. If, however, $S$ is any sector with central angle greater than $\frac{\pi}{2}$, then every non-trivial solution to $z^{-1} \mathfrak{u}^{\prime}=A(z) \mathfrak{u}$ tends to infinity as $z \rightarrow \infty$ on some sub-sector of $S$. This proves $\left(p_{1}, q_{1}\right)=\left(p_{2}, q_{2}\right)$ if $S$ is a half-plane.

REMARK. Theorem 14.1 in [11] applies, in particular, to algebraic systems

$$
t^{-n} \mathfrak{\mathfrak { y }}=A \mathfrak{y}+\mathfrak{f}(t, \mathfrak{y})
$$

$\left(\operatorname{det} A \neq 0,\|\mathfrak{f}(t, \mathfrak{y})\|=O\left(|t|^{-1}\right)+O\left(\|\mathfrak{y}\|^{2}\right)\right.$ as $\left.t \rightarrow+\infty,\|\mathfrak{y}\| \rightarrow 0\right)$ on arbitrary sectors with central angle $\frac{\pi}{n+1}$; the number $n+1 \geq 1$ is called rank. We note that it is also part of the hypothesis that the system has a formal solution $\sum_{k=1}^{\infty} \mathfrak{c}_{k} t^{-k}$, giving rise to the asymptotic solution $\mathfrak{y} \sim \sum_{k=1}^{\infty} \mathfrak{c}_{k} t^{-k}$; existence of the formal solution, however, follows immediately from $\operatorname{det} A \neq 0$.

5.7. Rational solutions. For rational solutions, the asymptotic series in (28) and (31) converge on $|z|>r_{0}$. Suppose that $p$ has $n^{\varrho}$ finite poles with residue $\varrho$. In the first case, integrating $p, q$, and $H$ over any large circle $|z|=r$ yields

$$
\sum_{\varrho^{3}=1} \varrho n^{\varrho}=-\alpha, \quad \sum_{\varrho^{3}=1}-\bar{\varrho} n^{\varrho}=-\beta \quad \text { and } \sum_{\varrho^{3}=1} n^{\varrho}=-\alpha \beta,
$$

hence $\beta=-\bar{\alpha}, \operatorname{Re} \alpha \in \frac{1}{2} \mathbb{Z}, \operatorname{Im} \alpha \in \frac{\sqrt{3}}{2} \mathbb{Z}$, and $-\alpha \beta=|\alpha|^{2} \in \mathbb{N}_{0}$. In the second case (31) we obtain in the same way, restricting ourselves to the case $\tau=1$ in (31),

$\sum_{\varrho^{3}=1} \varrho n^{\varrho}=\frac{1}{3}(\alpha+2 \beta+1), \quad \sum_{\varrho^{3}=1}-\varrho n^{\varrho}=\frac{1}{3}(2 \alpha+\beta-1), \sum_{\varrho^{3}=1} n^{\varrho}=\frac{1}{3}\left(\alpha^{2}+\beta^{2}+\alpha \beta-1\right)$,

hence again $\beta=-\bar{\alpha}, \operatorname{Re} \alpha \in 1+\frac{3}{2} \mathbb{Z}, \operatorname{Im} \alpha \in \frac{\sqrt{3}}{2} \mathbb{Z}$, and $\alpha^{2}+\bar{\alpha}^{2}-|\alpha|^{2}-1 \in 3 \mathbb{N}_{0}$.

\section{Solutions of minimal Growth}

6.1. The class $\mathfrak{P}_{\alpha, \beta}$. Our focus is on transcendental solutions $(p, q)$ to (1) satisfying $n(r, \Lambda) \asymp r^{2}$. These solutions form the class $\mathfrak{P}_{\alpha, \beta}$. If it is clear which parameters are in question or if the parameters are irrelevant, we shall write just $\mathfrak{P}$. By Theorem 5 , any solution that misses one kind of poles belongs to $\mathfrak{P}$, while from Theorem 4 it follows that either $\mathfrak{C}(p, q)=\left\{\frac{1}{3}\right\}$ or else $\mathfrak{C}(p, q)=\{0\}$ holds for $(p, q) \in \mathfrak{P}$. We also note that the union of classes $\mathfrak{P}_{\alpha, \beta}$ is invariant under Bäcklund transformations: $T(r, \mathrm{~B} p)=O(T(r, p))=O\left(r^{2}\right)$.

6.2. The case $\mathfrak{C}(\mathbf{p}, \mathbf{q})=\{\mathbf{0}\}$. In the first case we have

TheOREM 11. The solutions $(p, q)$ to equation (11) with cluster set $\mathfrak{C}(p, q)=\{0\}$ and counting function $n(r, \Lambda)=O\left(r^{2}\right)$ are rational functions, $g(z)=\exp \left(\int H(z) d z\right)$ is a polynomial and $p$ and $q$ are rational of degree $-\alpha \beta$.

Proof. There exist only finitely many strings of poles, and $p$ and $q$ have asymptotic expansions given by (28), one and the same on each sector $\left|\arg z-\nu \frac{\pi}{2}\right|<\frac{\pi}{4}$. From Theorem 9 it then follows that these expansions hold throughout the whole plane, hence $p$ and $q$ are rational functions, while the entire function $g$ satisfies $g(z) \sim C z^{-\alpha \beta}$ as $z \rightarrow \infty$, hence is a polynomial of degree $-\alpha \beta$. 
6.3. The case $\mathfrak{C}(\mathbf{p}, \mathbf{q})=\left\{\frac{\mathbf{1}}{3}\right\}$. For $(p, q) \in \mathfrak{P}_{\alpha, \beta}$ with $\mathfrak{C}(p, q)=\left\{\frac{1}{3}\right\}, p$ has $\mathfrak{n}^{\varrho}(p)$ strings of poles with residue $\varrho, \varrho^{3}=1$. It is quite natural to consider subclasses of $\mathfrak{P}_{\alpha, \beta}$ as follows: the pair $(p, q)$ belongs to class

$\mathfrak{P}_{\alpha, \beta}\left(\varrho_{1}\right), \quad$ if $\mathfrak{n}^{\varrho_{1}}(p)>0$, but $\mathfrak{n}^{\varrho_{2}}(p)=\mathfrak{n}^{\varrho_{3}}(p)=0\left(\varrho_{\nu}^{3}=1\right) ;$ $\mathfrak{P}_{\alpha, \beta}\left(\varrho_{1}, \varrho_{2}\right), \quad$ if $\mathfrak{n}^{\varrho_{1}}(p)>0$ and $\mathfrak{n}^{\varrho_{2}}(p)>0$, but $\mathfrak{n}^{\varrho_{3}}(p)=0$.

It is obvious that the six classes $\mathfrak{P}_{\alpha, \beta}(\varrho)$ and $\mathfrak{P}_{\alpha, \beta}\left(\varrho_{1}, \varrho_{2}\right)$ are mutually disjoint, and are contained in $\mathfrak{P}_{\alpha, \beta}$ by Theorem[5. We also note that $\mathfrak{n}^{\varrho}(p)=0$ implies that $p$ has at most finitely many poles with residue $\varrho$.

6.4. Asymptotics and the distribution of residues. Let $(p, q)$ be any pair in $\mathfrak{P}$ with cluster set $\mathfrak{C}(p, q)=\left\{\frac{1}{3}\right\}$. With each string of poles $s=\left(\lambda_{k}\right)$ we associate a polygon $\pi(s)$ with vertices $\lambda_{k}$. We assume that the polygons $\pi_{\nu}=\pi\left(s_{\nu}\right)$ are in cyclic order (counter-clockwise). They divide $|z|>r_{0}$ into finitely many domains $D_{1}, D_{2}, \ldots D_{n}\left(D_{n+1}=D_{1}\right) ; D_{\nu}$ is bounded by $\pi_{\nu}, \pi_{\nu+1}$ and some sub-arc of $|z|=r_{0}$. Re-scaling along any sequence $\left(\kappa_{m}\right)$ in $D_{\nu}$ with $\left|\kappa_{m}\right| \operatorname{dist}\left(\kappa_{m}, \Lambda\right) \rightarrow \infty$ yields constant limit functions $\mathfrak{p}=-\omega_{\nu}$ and $\mathfrak{q}=-\bar{\omega}_{\nu}$, hence

$$
p(z)=-\omega_{\nu} z+o(|z|), q(z)=-\bar{\omega}_{\nu} z+o(|z|) \quad\left(z \in D_{\nu},|z| \operatorname{dist}\left(z, \partial D_{\nu}\right) \rightarrow \infty\right) ;
$$

this does, of course, not mean that $p$ has an asymptotic expansion on $D_{\nu}$, except when $D_{\nu}$ contains some sector. To determine $\varrho_{\nu}=\operatorname{res}_{s_{\nu}} p$ we assume $\arg \lambda_{k} \sim 0$ for the sake of simplicity, and compute

$$
\frac{1}{2 \pi i} \int_{\gamma_{\nu}} p(z) d z=\varrho_{\nu} \frac{\sqrt{3}}{4 \pi} r^{2}+o\left(r^{2}\right)
$$

along a closed curve $\gamma_{\nu}$ surrounding $s_{\nu} \cap\left\{z: r_{0}<|z|<r\right\}$. Given $\delta>0$ sufficiently small, $\gamma_{\nu}$ consists of sub-arcs $\sigma_{r_{0}}$ and $\sigma_{r}$ of $|z|=r_{0}$ and $|z|=r$, of length at most $\delta r_{0}$ and $\delta r$, respectively, and $\operatorname{arcs} \gamma_{\nu-1}^{\prime}$ and $\gamma_{\nu}^{\prime}$ in $D_{\nu-1}$ and $D_{\nu}$, such that $p(z)=\omega_{\nu-1} z+o(|z|)$ and $p(z)=\omega_{\nu} z+o(|z|)$ hold as $z \rightarrow \infty$ on $\gamma_{\nu-1}^{\prime}$ and $\gamma_{\nu}^{\prime}$, respectively:

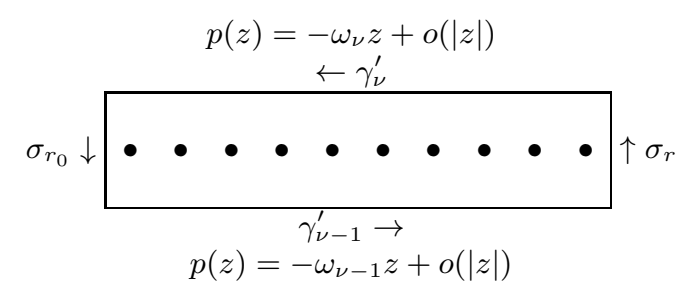

Figure 1 . The poles $\bullet$ have residue $\varrho_{\nu}$.

These arcs contribute to the integral (34) as follows:

$$
\begin{gathered}
\frac{1}{2 \pi i} \int_{\sigma_{r_{0}}} p(z) d z+\frac{1}{2 \pi i} \int_{\sigma_{r}} p(z) d z=O\left(\delta r^{2}\right) \\
\frac{1}{2 \pi i} \int_{\gamma_{\nu-1}^{\prime}} p(z) d z+\frac{1}{2 \pi i} \int_{\gamma_{\nu}^{\prime}} p(z) d z=\frac{-\omega_{\nu-1}+\omega_{\nu}}{i \sqrt{3}} \frac{\sqrt{3}}{4 \pi} r^{2}+O\left(\delta r^{2}\right),
\end{gathered}
$$


which implies $\varrho_{\nu}=i \frac{\omega_{\nu-1}-\omega_{\nu}}{\sqrt{3}}$. Table 1 displays the various possibilities (yes/no).

\begin{tabular}{|c|c|c|c|}
\hline$\omega_{\nu-1}$ & $\omega_{\nu}$ & $\varrho_{\nu}$ & $?$ \\
\hline 1 & $\omega$ & $-\bar{\omega}$ & no \\
\hline 1 & $\bar{\omega}$ & $\omega$ & yes \\
\hline$\omega$ & $\bar{\omega}$ & -1 & no \\
\hline$\omega$ & 1 & $\bar{\omega}$ & yes \\
\hline $\bar{\omega}$ & $\omega$ & 1 & yes \\
\hline $\bar{\omega}$ & 1 & $-\omega$ & no \\
\hline
\end{tabular}

$\arg p_{k} \sim 0 \bmod \pi$

\begin{tabular}{|c|c|c|c|}
\hline$\omega_{\nu-1}$ & $\omega_{\nu}$ & $\varrho_{\nu}$ & $?$ \\
\hline 1 & $\omega$ & $\bar{\omega}$ & yes \\
\hline 1 & $\bar{\omega}$ & $-\omega$ & no \\
\hline$\omega$ & $\bar{\omega}$ & 1 & yes \\
\hline$\omega$ & 1 & $-\bar{\omega}$ & no \\
\hline $\bar{\omega}$ & $\omega$ & -1 & no \\
\hline $\bar{\omega}$ & 1 & $\omega$ & yes \\
\hline
\end{tabular}

$\arg p_{k} \sim \frac{\pi}{2} \bmod \pi$

TABLE 1 . The asymptotics determines the residues, and vice versa $\left(\omega=\frac{1}{2}(-1+i \sqrt{3})\right)$.

6.5. Solutions of the first kind. Suppose that $(p, q) \in \mathfrak{P}_{\alpha, \beta}(1)$, say. Then $w=$ $p+q-z$ has only finitely many poles and vanishes at every pole with residue 1 . On the other hand it follows from $m(r, w)=O(\log r)$ that $w$ is a rational function, hence vanishes identically. From equation (3), (11), and also from the development of $p+q-z$ in (9) it then easily follows that $\alpha-\beta+1=0$ and $\mathbf{h}(\lambda)=0$. Conversely, let $(p, q)$ be any solution to (11) such that $p\left(z_{0}\right)+q\left(z_{0}\right)-z_{0}=0$ at some regular point. Then $w=p+q-z$ satisfies

$$
w^{\prime}=-q^{2}+p^{2}-z(p-q)-\alpha+\beta-1=(p(z)-q(z)) w \quad \text { and } \quad w\left(z_{0}\right)=0,
$$

hence vanishes identically $(p(z)-q(z)$ may be viewed as a coefficient).

TheOREM 12. The class $\mathfrak{P}_{\alpha, \beta}(\varrho)$ is non-empty if and only if $\varrho \alpha-\varrho \beta+1=0$. In that case, $(p, q) \in \mathfrak{P}_{\alpha, \beta}(\varrho)$ satisfies

$$
\bar{\varrho} p+\varrho q-z=0, \quad \mathbf{h}(\lambda)=0, \quad \text { and } \quad H=\frac{1}{3} z^{3}+\varrho \bar{\varrho} z+\bar{\varrho} p,
$$

and $p$ solves the Riccati equation

$$
p^{\prime}=-\alpha-\bar{\varrho} z^{2}+z p-\bar{\varrho} p^{2} .
$$

Proof. We restrict ourselves to the case $\varrho=1$. The differential equation (35) and the form of $H$ is obtained from (1) and (2) by replacing $q$ by $z-p$. To prove that $\mathfrak{P}_{\alpha, \alpha+1}(1)$ is non-empty, we start with any transcendental solution to equation (35) - not every solution can be rational - , and set $q=z-p$. Then $p^{\prime}=-q^{2}-z p-\alpha$ and $q^{\prime}=p^{2}+z q+\alpha+1$ immediately follow.

Remark. For $(p, q) \in \mathfrak{P}_{\alpha, \varrho \alpha+\bar{\varrho}}(\varrho)\left(\varrho=\frac{1}{2}(-1+i \sqrt{3})\right), w=p+q-z$ has simple poles with residue $\operatorname{res}_{\lambda} w=i \sqrt{3}$, and $w^{\prime}+\frac{w^{2}}{i \sqrt{3}}-i \sqrt{3} w$ is regular at $\lambda$ and assumes the value $\frac{1}{2}((3-i \sqrt{3}) \alpha-(3-i \sqrt{3}))$ at $z=\lambda$. This implies

$$
w^{\prime}=\frac{1}{2}((3-i \sqrt{3}) \alpha-(3-i \sqrt{3}))+i \sqrt{3} w-\frac{w^{2}}{i \sqrt{3}},
$$

and $w^{\prime}=\frac{i}{\sqrt{3}} w(w+3)$ if $\alpha=1$. In the latter case, $w$ has no zeros.

For $\varrho=1$, say, equation (35) may be transformed into

$$
u^{\prime}= \pm i \frac{2 \alpha+1}{\sqrt{3}}+\zeta^{2}-u^{2}
$$

(set $\zeta=a z$ and $p(z)-\frac{z}{2}=a u(\zeta)$ with $a^{4}=-\frac{3}{4}$ ). The transcendental solutions to equations of this type were analysed in [10, two major results may be described as follows: 
- The set of poles of generic solutions $u$ consist of four sequences $\left(\zeta_{n}^{(\nu)}\right)$ such that $\arg \zeta_{n}^{(\nu)} \sim(2 \nu-1) \frac{\pi}{4}$ and $\left|\zeta_{n}^{(\nu)}\right| \sim \sqrt{2 \pi n}$ as $n \rightarrow \infty$. Moreover, $u$ has an asymptotic expansion $u(\zeta)=(-1)^{\nu} \zeta+O\left(|\zeta|^{-1}\right)$ as $\zeta \rightarrow \infty$ on each open sector $\left|\arg \zeta-\nu \frac{\pi}{2}\right|<\frac{\pi}{4}$.

- There are four degenerate solutions $u_{\mu}$ having only sequences of poles for $\nu=\mu$ and $\nu=\mu+1$, such that $u_{\mu}(\zeta) \sim(-1)^{\mu} \zeta$ holds on $\left|\arg \zeta-\mu \frac{\pi}{2}\right|<\frac{\pi}{4}$, while $u_{\mu}(\zeta) \sim-(-1)^{\mu} \zeta$ holds on $2(\mu+1) \frac{\pi}{4}<\arg \zeta<(2 \mu+7) \frac{\pi}{4}$ (for some parameters $\alpha$, rational instead of degenerate solutions might occur).

In our case (35) it follows that $\Lambda$ consist of two or four strings $\left(\lambda_{k}\right)$, each with counting function $n\left(r,\left\{p_{k}\right\}\right) \sim \frac{\sqrt{3}}{4 \pi} r^{2}$ and asymptotic to the rays $\arg z=\nu \frac{\pi}{2}(\nu=$ $\mu, \mu+1$ or $\nu=0,1,2,3)$. From Table 1 it follows that the residues determine the asymptotics.

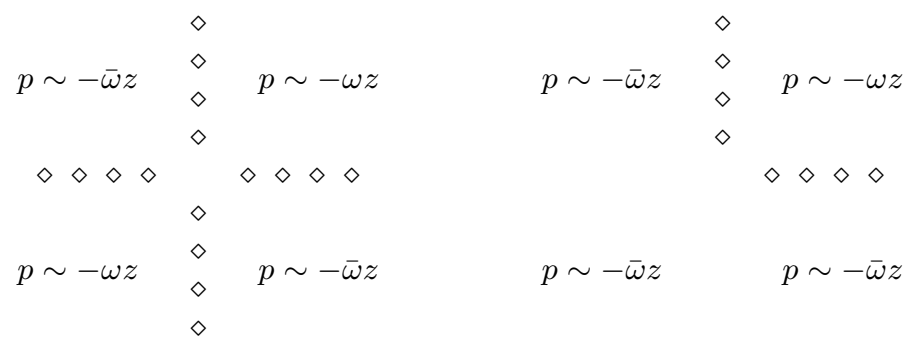

FigURE 2. Generic and degenerate distribution of poles and asymptotics in $\mathfrak{P}_{\alpha, \alpha+1}(1)$; each symbol $\diamond$ represents a pole with residue $1 ; \omega=\frac{1}{2}(-1+i \sqrt{3})$.

6.6. Solutions of the second kind. We will confine ourselves to the symmetric class $\mathfrak{P}_{\alpha, \beta}(\varrho, \bar{\varrho})$, noting that $\mathrm{M}_{\bar{\varrho}} \mathfrak{P}_{\alpha, \beta}(1, \varrho)=\mathfrak{P}_{\varrho \alpha, \bar{\varrho} \beta}(\varrho, \bar{\varrho})$. For $\omega \in\{\varrho, \bar{\varrho}\}$ we consider the linear combination $u_{\omega}=\omega p+\bar{\omega} q+\frac{z}{2} ; u_{\omega}$ is regular at poles of $p$ with residue $\bar{\omega}$ and has simple poles at poles of $p$ with residue $\omega$ :

$$
\begin{aligned}
u_{\omega}(z) & =\frac{-2 i \operatorname{Im} \omega}{z-\lambda}+a(\lambda ; \omega)(z-\lambda)+\cdots \\
a(\lambda ; \omega) & =-\frac{1}{2}+\frac{1}{3}[(\omega+2) \alpha-(\bar{\omega}+2) \beta]+\frac{i}{2}(\operatorname{Im} \omega) \lambda^{2} .
\end{aligned}
$$

Like in the first case we may conclude that $u_{\omega}$ satisfies the Riccati equation

$$
u_{\omega}^{\prime}=3 a(z ; \omega)+\frac{u_{\omega}^{2}}{2 i \operatorname{Im} \omega} .
$$

This means, in particular, that $p$ has four [two] strings of poles with residues $\varrho$ and $\bar{\varrho}$, asymptotic to all [two adjacent] directions $\arg z=\nu \frac{\pi}{2}$. Combining (11) and (37) yields an algebraic equation $K_{\omega}(z, p, q)=0$, and the system $\left(K_{\varrho}=0, K_{\bar{\varrho}}=0\right)$ has resultant $-\frac{16}{3}(\alpha-\beta-2)^{2}$ with respect to each of $p$ and $q$, thus

$$
-\alpha+\beta+2=0
$$

and also

$$
K(z, p, q)=p^{2}+q^{2}+z^{2}-p q+z(p+q)+3 \beta+3 \equiv 0
$$

is necessary for $\mathfrak{P}_{\alpha, \beta}(\varrho, \bar{\varrho}) \neq \emptyset$. We also note that $(\alpha, \beta)=(1,-1)$ is the unique solution to the linear system $-\alpha+\beta+2=0, \bar{\omega} \alpha-\omega \beta+1=0$. By Proposition 1 below we obtain $(\alpha, \beta) \neq(1,-1)$ as further necessary condition for $\mathfrak{P}_{\alpha, \beta}(\varrho, \bar{\varrho}) \neq \emptyset$. Based on these considerations we will prove: 
TheOrem 13. The class $\mathfrak{P}_{\alpha, \beta}(\varrho, \bar{\varrho})$ with $(\alpha, \beta) \neq(1,-1)$ is non-empty if and only if (38) holds. The Bäcklund transformation $\mathrm{B}_{\varrho}$ maps $\mathfrak{P}_{\beta+2, \beta}(\varrho, \bar{\varrho})$ into $\mathfrak{P}_{\varrho \beta-\bar{\varrho}}, \bar{\varrho} \beta+\bar{\varrho}-1(\varrho)$. Moreover,

$$
H(z, p, q)=q-p+\frac{1}{3} z^{3}+(\beta+1) z
$$

and $\mathbf{h}(\lambda)+i \operatorname{Im} \omega=(\beta+1) \lambda\left(\operatorname{res}_{\lambda} p=\omega \in\{\varrho, \bar{\varrho}\}\right)$ hold.

Proof. Replacing $p q, q^{2}+z p$, and $p^{2}+z q$ in (39) by $H^{\prime},-p^{\prime}-2-\beta$, and $q^{\prime}-\beta$, respectively, we obtain $(q-p-H)^{\prime}+z^{2}+\beta+1=0$, hence also

$$
H(z, p, q)=q-p+\frac{1}{3} z^{3}+(\beta+1) z+c
$$

holds. Since $p$ and $q$ are transcendental, this is compatible with (39) if and only if $c=0$ (we note that $\frac{1}{3}\left(p^{3}+q^{3}-z^{3}\right)+z p q=\frac{1}{3}(p+q-z)\left(p^{2}+q^{2}+z^{2}-p q+z(p+\right.$ $q)+2 \beta+2)$ ). Thus also (40) holds, while $\mathbf{h}(\lambda)+i \operatorname{Im} \omega=(\beta+1) \lambda$ at poles of $p$ with residue $\omega \in\{\varrho, \bar{\varrho}\}$ follows from (37) and (8).

To prove the second assertion, we note that $(\alpha, \beta) \neq(1,-1)$ and (38) imply that $\varrho \alpha-\bar{\varrho} \beta+1 \neq 0$, and also that the function $\varrho p+\varrho q-z$ does not vanish identically since it has poles at poles of $p$ with residue $\varrho$. According to (13), the poles of $p$ with residue $\varrho$ survive as poles of $\mathrm{B}_{\varrho} p$ with the same residue, while the poles of $p$ with residue $\bar{\varrho}$ disappear (we note that $\mathfrak{n}^{\varrho}(p)=\mathfrak{n}^{\bar{\varrho}}(p)$ ). The zeros of $f=\varrho p+\bar{\varrho} q-z$ which are not poles of $p$ with residue $\bar{\varrho}$ may create poles with residue $\bar{\varrho}$. From $T(r, f)=$ $N(r, f)+O(\log r)=N\left(r, \Lambda^{\varrho}\right)+O(\log r)=N\left(r, \Lambda^{\bar{\varrho}}\right)+o\left(r^{2}\right)$ and Nevanlinna's First Main Theorem, however, it follows that

$$
N\left(r, \Lambda^{\bar{\varrho}}\right) \leq N(r, 1 / f) \leq T(r, f)+O(1)=N\left(r, \Lambda^{\varrho}\right)+O(\log r)=N\left(r, \Lambda^{\bar{\varrho}}\right)+o\left(r^{2}\right),
$$

hence $f$ has at most $o\left(r^{2}\right)$ zeros on $|z|<r$ except those arising from poles of $p$ with residue $\bar{\varrho}$. This proves $\mathfrak{n}^{\bar{\varrho}}\left(\mathrm{B}_{\varrho} p\right)=\mathfrak{n}^{1}\left(\mathrm{~B}_{\varrho} p\right)=0$ and $\mathrm{B}_{\varrho}(p, q) \in \mathfrak{P}_{\varrho} \beta-\bar{\varrho}, \bar{\varrho} \beta+\bar{\varrho}-1(\varrho)$.

To prove that (38) together with $(\alpha, \beta) \neq(1,-1)$ is sufficient for $\mathfrak{P}_{\alpha, \beta}(\varrho, \bar{\varrho}) \neq \emptyset$, we start with any transcendental pair $(\tilde{p}, \tilde{q}) \in \mathfrak{P}_{\tilde{\alpha}, \tilde{\beta}}(\varrho)$ with $(\tilde{\alpha}, \tilde{\beta})=(\varrho \beta-\bar{\varrho}, \bar{\varrho} \beta+\bar{\varrho}-1)$, and note that $\bar{\varrho} \tilde{\alpha}-\varrho \tilde{\beta}+1=\beta-\varrho-(\beta+1-\varrho)+1=0$, but $\varrho \tilde{\alpha}-\bar{\varrho} \tilde{\beta}+1=$ $(\bar{\varrho}-\varrho) \beta-1-(\varrho-\bar{\varrho})+1 \neq 0$ for $\beta \neq-1$. Thus $\varrho \tilde{p}+\varrho p-z$ vanishes identically, but $\varrho \tilde{p}+\varrho \bar{q}-z$ does not, so that $(p, q)=\mathrm{B}_{\varrho}(\tilde{p}, \tilde{q})$ is well defined. From (13) it then follows that the poles of $\tilde{p}$ remain poles of $p$ with residue $\varrho$, while new poles with residue $\bar{\varrho}$, but none with residue 1 , might be created by the zeros of $\tilde{f}=\varrho \tilde{p}+\bar{\varrho} p-z$, if any. Since $(p, q)$ solves (11) with parameter $(\beta+2, \beta)$, hence $(\beta+2)-\beta+1 \neq 0$, the poles with residue $\bar{\varrho}$ actually exist, and $(p, q) \in \mathfrak{P}_{\beta+2, \beta}(\varrho, \bar{\varrho})$ follows.

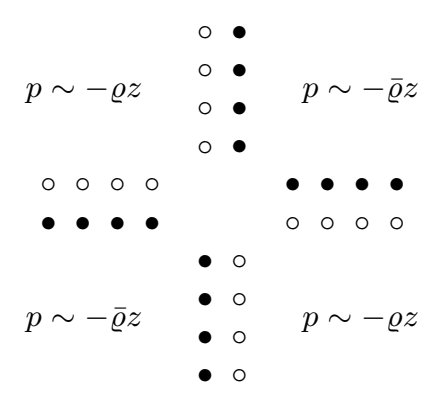

FiguRE 3. Distribution of poles and asymptotics in $\mathfrak{P}(\varrho, \bar{\varrho})$, res $\bullet=\varrho=\frac{1}{2}(-1+i \sqrt{3})$, res。 $p=\bar{\varrho}$; in the narrow domains between two strings, $p(z)=-z+o(|z|)$ holds. 
REMARK. The case $(\alpha, \beta)=(1,-1)$ is indeed exceptional: $\mathfrak{P}_{1,-1}(\varrho, \bar{\varrho})$ is empty as follows from Proposition 11 below with $\varrho_{1}=\varrho$ and $\varrho_{1}=\bar{\varrho}$, respectively. We also note that in this case, (11) admits polynomial solutions $p=q=-z$.

\section{SOlutions OF THE THIRD KIND}

7.1. Preliminary remarks. Functions $(p, q) \in \mathfrak{P}_{\alpha, \beta}$ have cluster set $\mathfrak{C}(p, q)=\left\{\frac{1}{3}\right\}$ and finitely many strings of poles, each asymptotic to some ray $\arg z=\nu \frac{\pi}{2}$ and with counting function $\sim \frac{\sqrt{3}}{4 \pi} r^{2}$, so that

$$
n(r, \Lambda) \sim \mathfrak{n}(p) \frac{\sqrt{3}}{4 \pi} r^{2}, \quad n\left(r, \Lambda^{\varrho}\right) \sim \mathfrak{n}^{\varrho}(p) \frac{\sqrt{3}}{4 \pi} r^{2} \quad \text { and } \quad \sum_{\varrho^{3}=1} \mathfrak{n}^{\varrho}(p)=\mathfrak{n}(p) .
$$

Moreover, $p$ and $q$ have asymptotic expansions $p(z) \sim-\tau_{\nu} z$ and $q(z) \sim-\bar{\tau}_{\nu} z$ on the quarter-planes $(\nu-1) \frac{\pi}{2}<\arg z<\nu \frac{\pi}{2}(1 \leq \nu \leq 4)$. This will be abbreviated by writing

$$
p: \begin{array}{l|l}
\tau_{2} & \tau_{1} \\
\hline \tau_{3} & \tau_{4}
\end{array}
$$

7.2. Special cases. Before proceeding further we will examine two special cases:

Proposition 1. Suppose that $(p, q) \in \mathfrak{P}_{\alpha, \beta}$ satisfies

$$
\bar{\varrho}_{1} \alpha-\varrho_{1} \beta+1=0 \quad \text { and } \quad \mathfrak{n}^{\varrho_{1}}(p)>\frac{1}{2}\left(\mathfrak{n}^{\varrho_{2}}(p)+\mathfrak{n}^{\varrho_{3}}(p)\right) .
$$

Then $(p, q) \in \mathfrak{P}_{\alpha, \beta}\left(\varrho_{1}\right)$, that is, $\bar{\varrho}_{1} p+\varrho_{1} q-z \equiv 0$.

Proof. We may assume that $\varrho_{1}=1$, hence $\alpha-\beta+1=0$ and $\mathfrak{n}^{1}(p)>\frac{1}{2}\left(\mathfrak{n}^{\varrho}(p)+\mathfrak{n}^{\bar{\varrho}}(p)\right)$ with $\varrho=\frac{1}{2}(-1+\sqrt{3})$ hold. Then $w=p+q-z$ vanishes at least twice at poles $\lambda \in \Lambda^{1}$, and if we assume that $w$ does not vanish identically, it follows from $m(r, w)=$ $O(\log r)$ and Nevanlinna's First Main Theorem that

$$
2 N\left(r, \Lambda^{1}\right) \leq N(r, 1 / w) \leq T(r, w)+O(1)=N\left(r, \Lambda^{\varrho}\right)+N\left(r, \Lambda^{\bar{\varrho}}\right)+O(\log r),
$$

hence also $2 \mathfrak{n}^{1}(p) \leq \mathfrak{n}^{\varrho}(p)+\mathfrak{n}^{\bar{\varrho}}(p)$ against our hypothesis, and Proposition 1 is proved completely.

Proposition 2. For $(p, q) \in \mathfrak{P}_{\alpha, \beta}$ with signature 411), the sum $\sum_{\nu=1}^{4}(-1)^{\nu} \tau_{\nu}$ is non-zero.

Proof. Obviously, $\sum_{\nu=1}^{4}(-1)^{\nu} \tau_{\nu}=0$ is only possible if, up to some rotation of the plane and up to replacing $(p, q)$ by $\mathrm{M}_{\varrho}(p, q)$ with $\varrho$ suitably chosen, $(p, q)$ has the signature $\frac{\tau}{\bar{\tau}} \mid \bar{\tau}$ with $\tau=\frac{1}{2}(-1+i \sqrt{3})$. From Theorem 7 it then follows that

$$
H(z)-\frac{z^{3}}{3} \sim \begin{cases}-(\tau \alpha+\bar{\tau} \beta) z+\frac{\tau \alpha^{2}+\bar{\tau} \beta^{2}+\alpha \beta-1}{3 z}+\cdots & (\operatorname{Im} z>0) \\ -(\bar{\tau} \alpha+\tau \beta) z+\frac{\bar{\tau} \alpha^{2}+\tau \beta^{2}+\alpha \beta-1}{3 z}+\cdots & (\operatorname{Im} z<0)\end{cases}
$$

holds. The entire function $g(z)=\exp \left(\int H(z) d z-\frac{z^{4}}{12}\right)$ has order of growth at most four and satisfies

$$
g(z)=\left\{\begin{array}{ll}
A e^{a z^{2}} z^{\mu}(1+o(1)) & \left(z=r e^{i \delta} \rightarrow \infty\right) \\
B e^{b z^{2}} z^{\nu}(1+o(1)) & \left(z=r e^{-i \delta} \rightarrow \infty\right)
\end{array} \quad(0<\delta<\pi)\right.
$$


with $A B \neq 0, a=-\frac{1}{2}(\tau \alpha+\bar{\tau} \beta), b=-\frac{1}{2}(\bar{\tau} \alpha+\tau \beta), \mu=\frac{1}{3}\left(\tau \alpha^{2}+\bar{\tau} \beta^{2}+\alpha \beta-1\right)$ and $\nu=\frac{1}{3}\left(\bar{\tau} \alpha^{2}+\tau \beta^{2}+\alpha \beta-1\right)$.

In the first step we will show that $\operatorname{Re} a=\operatorname{Re} b$, and assume to the contrary that $\operatorname{Re} a>\operatorname{Re} b$. Then $f(z)=e^{-a z^{2}} z^{-\mu} g(z)$ satisfies

$$
f\left(r e^{i \delta}\right) \rightarrow A \neq 0 \text { and }\left|f\left(r e^{-i \delta}\right)\right|=O\left(e^{(\operatorname{Re} b-\operatorname{Re} a) r^{2} \cos 2 \delta} r^{M}\right) \rightarrow 0
$$

as $r \rightarrow \infty$, which for $0<\delta<\delta_{0}$ sufficiently small contradicts the PhragménLindelöf Principle. Thus Re $a \leq \operatorname{Re} b$ holds, and in the same manner (consider $\overline{f(\bar{z})}$ instead of $f$ with $(a, b)$ replaced by $(\bar{b}, \bar{a})$ we obtain $\operatorname{Re} b \leq \operatorname{Re} a$. The same argument will now show that $\operatorname{Im} a \leq \operatorname{Im} b$ : assuming to the contrary that $(\operatorname{Re} a=\operatorname{Re} b)$ and $\operatorname{Im} a>\operatorname{Im} b$ holds, it follows that

$$
f\left(r e^{i \delta}\right) \rightarrow A \neq 0 \quad \text { and } \quad\left|f\left(r e^{-i \delta}\right)\right|=O\left(e^{-(\operatorname{Im} a-\operatorname{Im} b) r^{2} \sin 2 \delta} r^{M}\right) \rightarrow 0 \quad(r \rightarrow \infty),
$$

which for $\delta>0$ sufficiently small again contradicts the Phragmén-Lindelöf Principle. Moreover, $\operatorname{Im} a=\operatorname{Im} b$ implies that $f$ has only finitely many zeros, and $p$ has only finitely many poles asymptotic to the positive real axis. The same argument, however, works for $g(-z)$ on $|\arg z|<\delta_{0}$, with $(a, b)$ replaced by $(b, a)$, this showing that also $\operatorname{Im} b \leq \operatorname{Im} a$ holds. In particular, we have $a=b$, this implying that $g$ has only finitely many zeros at all, and $p$ has only finitely many poles in contrast to our assumption that $p$ is transcendental. Thus Proposition 2 is completely proved.

7.3. The class $\mathfrak{P}_{\alpha, \beta}$. We will now prove the main result of this section:

TheOREm 14. To any $(p, q) \in \mathfrak{P}_{\alpha, \beta}$ there exists some Bäcklund transformation $\mathrm{B}$, such that

$$
\mathrm{B} p+\mathrm{B} q-z=0 \quad \text { and } \quad \mathrm{B} \alpha-\mathrm{B} \beta+1=0
$$

holds, that is, $\mathrm{B}(p, q) \in \mathfrak{P}_{\mathrm{B} \alpha, \mathrm{B} \alpha+1}(1)$.

Proof. Suppose $(p, q) \in \mathfrak{P}_{\alpha, \beta}$ has signature (41). On combination with the asymptotics of $p$, Cauchy's Theorem gives

$$
\sum_{\varrho^{3}=1} \varrho \mathfrak{n}^{\varrho}(p)=2 i \sum_{\nu=1}^{4}(-1)^{\nu} \frac{\tau_{\nu}}{\sqrt{3}}
$$

Taking real and imaginary parts, we obtain

$$
\begin{aligned}
\mathfrak{n}^{1}(p)-\frac{1}{2}\left(\mathfrak{n} \varrho(p)+\mathfrak{n}^{\bar{\varrho}}(p)\right) & =-2 \sum_{\nu=1}^{4}(-1)^{\nu} \frac{\operatorname{Im} \tau_{\nu}}{\sqrt{3}} \\
\frac{1}{2}\left(\mathfrak{n}^{\varrho}(p)-\mathfrak{n}^{\bar{\varrho}}(p)\right) & =2 \sum_{\nu=1}^{4}(-1)^{\nu} \frac{\operatorname{Re} \tau_{\nu}}{3}
\end{aligned} \quad\left(\varrho=\frac{1}{2}(-1+i \sqrt{3})\right) .
$$

By Proposition 2 we have $\mathfrak{n}^{\varrho_{1}}(p)>\frac{1}{2}\left(\mathfrak{n}^{\varrho_{2}}(p)+\mathfrak{n}^{\varrho_{3}}(p)\right)$ for some $\varrho_{1}$. We may assume $\varrho_{1}=1$, hence

$$
\mathfrak{n}^{1}(p)>\frac{1}{2}\left(\mathfrak{n}^{\varrho}(p)+\mathfrak{n}^{\bar{\varrho}}(p)\right)>0
$$

otherwise we would replace $(p, q)$ by $\mathrm{M}_{\varrho_{1}}(p, q)=\left(\bar{\varrho}_{1} p, \varrho_{1} q\right)$, but retain the notation $(p, q)$ and $(\alpha, \beta)$. By Proposition 1 we are done if $\alpha-\beta+1=0$. Otherwise we apply $B_{1}$ to obtain

$$
\tilde{p}(z)=p(z)-\frac{\alpha-\beta+1}{p(z)+q(z)-z}, \tilde{q}(z)=q(z)+\frac{\alpha-\beta+1}{p(z)+q(z)-z}
$$


Under $\mathrm{B}_{1}$, the Hamiltonian and the signature change as follows:

$$
\tilde{H}(z)-H(z)=-\frac{\alpha-\beta+1}{p(z)+q(z)-z}=\tilde{p}(z)-p(z)=-\tilde{q}(z)+q(z)
$$

and

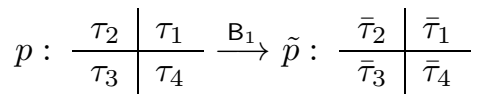

Although $H$ and $\tilde{H}$ cannot be controlled sufficiently well close to the rays $\arg z=$ $\nu \frac{\pi}{2}$, this can be done for $\tilde{H}(z)-H(z)$ to compute

$$
n(r, \tilde{p})-n(r, p)=\frac{1}{2 \pi i} \int_{\Gamma_{r}}(\tilde{H}(z)-H(z)) d z=\frac{1}{2 \pi i} \int_{\Gamma_{r}}(\tilde{p}(z)-p(z)) d z
$$

asymptotically (it is obvious that the curve $\Gamma_{r}$, constructed in section 4 may be modified in such a way that $|p(z)|+|\tilde{p}(z)|=O(|z|)$ holds on $\left.\Gamma_{r}\right)$. From

$$
\tilde{p}(z)-p(z)=2 i\left(\operatorname{Im} \tau_{\nu}\right) z+O\left(|z|^{-1}\right)
$$

uniformly on $\left|\arg z-\left(\nu-\frac{1}{2}\right) \pi\right|<\frac{\pi}{4}-\delta$, and (43) it follows that

$$
\mathfrak{n}(\tilde{p})-\mathfrak{n}(p)=4 \sum_{\nu=1}^{4}(-1)^{\nu} \frac{\operatorname{Im} \tau_{\nu}}{\sqrt{3}}=-2 \mathfrak{n}^{1}(p)+\mathfrak{n}^{\varrho}(p)+\mathfrak{n}^{\bar{\varrho}}(p) \leq-1
$$

Repeating this argument yields a sequence $\left(\mathrm{B}^{[k]}\right)$ of Bäcklund transformations $\mathrm{B}^{[k]}=\mathrm{B}_{1} \mathrm{M}_{\varrho^{[k-1]}} \mathrm{B}^{[k-1]}$, such that, for some $n$,

$$
\mathrm{B}^{[n]} p+\mathrm{B}^{[n]} q-z \equiv 0 \quad \text { and } \quad \mathrm{B}^{[n]} \alpha-\mathrm{B}^{[n]} \beta+1=0 .
$$

7.4. Concluding remark. Since the Bäcklund transformations do not change the number of different $\tau_{\nu}$ 's, and the functions in $\mathfrak{P}_{\alpha, \beta}(\omega)$ have only signatures with two different $\tau_{\nu}$ 's, this is also true for any $(p, q) \in \mathfrak{P}$, and will simplify matter considerably. Up to a rotation of the plane, there are two possibilities: \begin{tabular}{l|l}
$\tau_{2}$ & $\tau_{1}$ \\
\hline$\tau_{1}$ & $\tau_{2}$
\end{tabular} (generic) and \begin{tabular}{l|l}
$\tau_{2}$ & $\tau_{1}$ \\
\hline$\tau_{2}$ & $\tau_{2}$
\end{tabular} (degenerate). We will discuss in detail the case $\mathfrak{n}^{1} \leq \mathfrak{n}^{\bar{\varrho}} \leq \mathfrak{n}^{\varrho}$ $\left(\varrho=\frac{1}{2}(-1+i \sqrt{3})\right.$ ), which by (42) is equivalent to $\operatorname{Im} \tau_{1} \leq \operatorname{Im} \tau_{2}$ and $\operatorname{Re} \tau_{1} \leq \operatorname{Re} \tau_{2}$, but $\tau_{1} \neq \tau_{2}$. The case $\mathfrak{n}^{1}=\mathfrak{n}^{\varrho}=\mathfrak{n}^{\bar{\varrho}}$ is excluded by Proposition 2, There are two (non-degenerate) kinds of solutions:

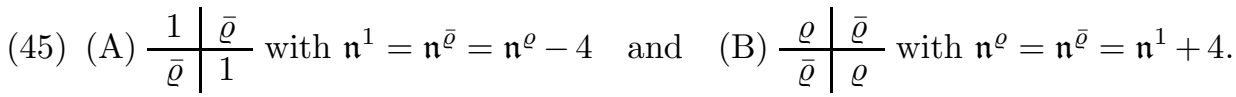


Based on Table 1, it is easy to show that, in first non-trivial cases, the residues are distributed as follows:

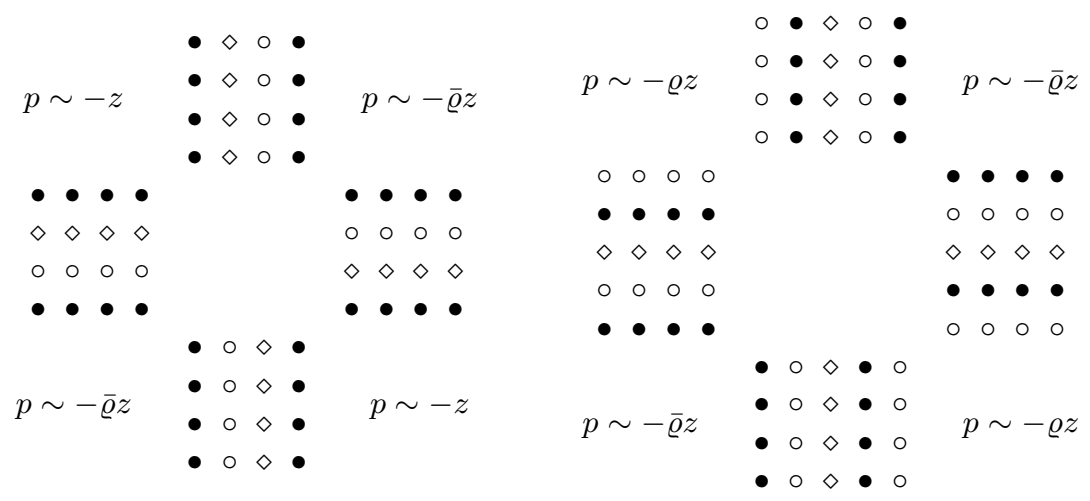

Figure 4. Case $(\mathrm{A}): \mathfrak{n}^{\varrho}=8, \mathfrak{n}^{\bar{\varrho}}=\mathfrak{n}^{1}=4$, and case $(\mathrm{B}): \mathfrak{n}^{\varrho}=\mathfrak{n}^{\bar{\varrho}}=8, \mathfrak{n}^{1}=4$.

\section{Application to fourth Painlevé transcendents}

8.1. Fourth Painlevé transcendents with positive deficiency $\delta(0, w)$. It is well-known and easy to prove that any transcendental solution to equation (3) assumes every value $c \neq 0$ equally often in the sense that $m(r, 1 /(w-c))=O(\log r)$. This is also true if $c=0$, but $\alpha-\beta+1 \neq 0$. In case of $\alpha-\beta+1=0$ there are, however, solutions with Picard value zero; they satisfy $T(r, w)=O\left(r^{2}\right)$. Ignoring this case, it is only known that $m(r, 1 / w) \leq \frac{1}{2} T(r, w)+O(\log r)$ holds (equivalently, $m(r, 1 / y) \leq \frac{1}{2} T(r, y)+O(\log r)$, see [2, 7] $)$. We will prove the following surprising and never expected result:

THEOREM 15. Let $w=p+q-z$ be any transcendental solution to (3) with positive Nevanlinna deficiency $\delta(0, w)=1-\limsup _{r \rightarrow \infty} \frac{N(r, 1 / w)}{T(r, w)}$. Then $(p, q)$ belongs to $\mathfrak{P}_{\alpha_{k}, \alpha_{k}+1}$ with $\alpha_{k}=\frac{1}{2}(-1 \pm(2 k+1) \sqrt{3} i), k \in \mathbb{N}_{0}$, and $\delta(0, w)=\frac{1}{2 k+1}$. For $k=0$, $w$ satisfies the Riccati equation $w^{\prime}=\frac{\mp i}{\sqrt{3}}\left(3 z w+w^{2}\right)$, and zero is a Picard value.

Proof. Since $w=p+q-z$ vanishes at least twice at poles of $p$ with residue 1 , Theorem 5 yields

$$
T(r, w)=\frac{2}{3} N(r, \Lambda)+O\left(r^{2}\right)=2 N\left(r, \Lambda^{1}\right)+O\left(r^{2}\right) \leq N(r, 1 / w)+O\left(r^{2}\right) .
$$

This proves $\delta(0, w)=0$ provided $\limsup _{r \rightarrow \infty} T(r, w) / r^{2}=\infty$. Otherwise $T(r, w)=$ $O\left(r^{2}\right)$ holds, and $(p, q)$ belongs to the class $\mathfrak{P}_{\alpha, \alpha+1}$ for some $\alpha$. From (11) and $\alpha-\beta+1=0$ it follows that $w^{\prime} / w=p-q$, hence $w$ has zeros of order two at the poles of $p$ with residue 1 , and no others. In any case we have

$$
1-\delta(0, w)=\frac{2 \mathfrak{n}^{1}(p)}{\mathfrak{n} \varrho(p)+\mathfrak{n}^{\bar{\varrho}}(p)},
$$

and $\delta(0, w)>0$ implies $\mathfrak{n}^{1}(p)<\frac{1}{2}\left(\mathfrak{n}^{\varrho}(p)+\mathfrak{n}^{\bar{\varrho}}(p)\right)$. It suffices to discuss the signatures (45), thus to assume $\mathfrak{n}^{\varrho} \geq \mathfrak{n}^{\bar{\varrho}}$ and $\mathfrak{n}^{\varrho}>\mathfrak{n}^{1}$. In both cases, the proof of Theorem 14 shows that the sequence

$$
(p, q) \stackrel{\mathrm{M}_{\varrho}}{\longrightarrow}\left(p_{1}, q_{1}\right) \stackrel{\mathrm{B}_{1}}{\longrightarrow}\left(p_{2}, q_{2}\right) \stackrel{\mathrm{M}_{\varrho}}{\longrightarrow}\left(p_{3}, q_{3}\right) \stackrel{\mathrm{B}_{1}}{\longrightarrow}\left(p_{4}, q_{4}\right) \stackrel{\mathrm{M}_{\varrho}}{\longrightarrow}\left(p_{5}, q_{5}\right) \cdots
$$


terminates at $\left(p_{n}, q_{n}\right) \in \mathfrak{P}_{\alpha_{n}, \alpha_{n}+1}(1)$. We obtain

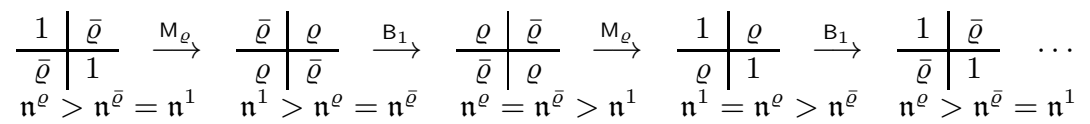

and

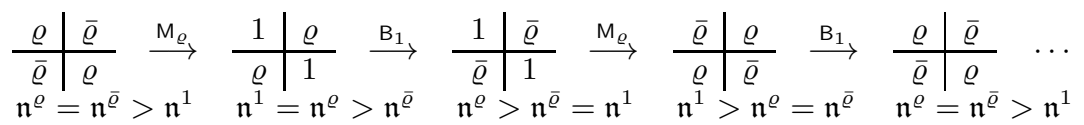

respectively, and $\alpha_{4 k}=\alpha+k \varrho-k, \beta_{4 k}=\beta-k \bar{\varrho}+k$, hence $\alpha_{4 k+1}=\bar{\varrho} \alpha+k-k \bar{\varrho}$, $\beta_{4 k+1}=\varrho \beta-k+k \varrho, \alpha_{4 k+3}=\beta-(k+1) \varrho+k, \beta_{4 k+3}=\alpha+(k+1) \varrho-k$, and

$$
\begin{aligned}
& \alpha_{4 k+1}-\beta_{4 k+1}+1=\varrho \alpha-\varrho \beta+1+3 k \\
& \alpha_{4 k+3}-\beta_{4 k+3}+1=-(\alpha-\beta+1)+3 k+3 .
\end{aligned}
$$

in both cases. The procedure stops when $\mathfrak{n}^{\varrho}=\mathfrak{n}^{\bar{\varrho}}=0$, this implying $n=4 k+3$ in case (B). Then, however, $\alpha-\beta+1=0$ is impossible. In case (A) the procedure stops when $n=4 k+1$. Then $\alpha-\beta+1=\alpha_{4 k+1}-\beta_{4 k+1}+1=0$ has the unique solution $\alpha=-\frac{1}{2}(1+(2 k+1) \sqrt{3} i), \beta=\alpha+1$. In case of $k=0(\alpha=\bar{\varrho}), w$ satisfy the Riccati equation with the upper sign, and has Picard value zero, and in any case the procedure may be reversed. It suffices to describe the first non-trivial step $(k=1)$ : we start with any generic $\left(p_{0}, q_{0}\right) \in \mathfrak{P}_{\alpha_{0}, \alpha_{0}+1}(1)$ with $\alpha_{0}=\frac{1}{2}(-1+3 \sqrt{3} i)$ and $\left(\mathfrak{n}^{1}, \mathfrak{n}^{\varrho}, \mathfrak{n}^{\bar{\varrho}}\right)=(4,0,0)$, and consider the sequence

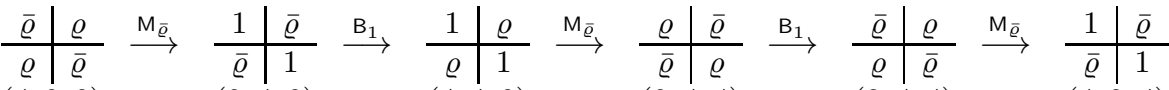

$$
\begin{aligned}
& (4,0,0) \quad(0,4,0) \quad(4,4,0) \quad(0,4,4) \quad(8,4,4) \quad(4,8,4)
\end{aligned}
$$

Then $w=p+q-z$ with $(p, q)=\mathrm{M}_{\bar{\varrho}} \mathrm{B}_{1} \mathrm{M}_{\bar{\varrho}} \mathrm{B}_{1} \mathrm{M}_{\bar{\varrho}}\left(p_{0}, q_{0}\right) \in \mathfrak{P}_{-\frac{1}{2}(1+3 \sqrt{3} i), \frac{1}{2}(1-3 \sqrt{3} i)}$ has deficiency

$$
\delta(0, w)=\frac{\mathfrak{n}^{\varrho}+\mathfrak{n}^{\bar{\varrho}}-2 \mathfrak{n}^{1}}{\mathfrak{n}^{\varrho}+\mathfrak{n}^{\bar{\varrho}}}=\frac{8+4-2 \cdot 4}{8+4}=\frac{1}{3} .
$$

In the next step $\left(k=2\right.$ and $\left.\alpha_{0}=\frac{1}{2}(-1+5 \sqrt{3} i)\right)$ we would land at $(p, q) \in$ $\mathfrak{P}_{-\frac{1}{2}(1+5 \sqrt{3} i), \frac{1}{2}(1-5 \sqrt{3} i)}$ with $\mathfrak{n}^{\varrho}=12, \mathfrak{n}^{\bar{\varrho}}=\mathfrak{n}^{1}=8$ and $\delta(0, w)=\frac{1}{5}$ etc. This is also true in the degenerate cases.

REMARK. It is not hard to prove that the exceptional solutions satisfy some implicit first order algebraic differential equation $P\left(z, w, w^{\prime}\right)=0$ of degree $2 k+1$ with respect to $w^{\prime}$. The corresponding parameters in (4) are $\hat{\alpha}=2 k+1$ and $\hat{\beta}=0$.

8.2. An open problem. The transcendental solutions to (11) as well as to (3) and (44) have Nevanlinna characteristic bounded below by $C_{1} r^{2}$ and above by $C_{2} r^{4}$. It is still open whether or not either $T(r, p) \asymp r^{2}$ or else $T(r, p) \asymp r^{4}$ holds.

8.3. Acknowledgement. I would like to thank the referee for his helpful comments.

\section{REFERENCES}

[1] L. Ahlfors, Complex analysis, McGraw-Hill 1979.

[2] V. Gromak, I. Laine, and S. Shimomura, Painlevé Differential Equations in the Complex Plane, W. de Gruyter, Berlin-New York, 2002.

[3] W. Hayman, Meromorphic functions, Oxford Clarendon Press 1964.

[4] Th. Kecker, A cubic polynomial Hamiltonian system with meromorphic solutions, preprint 2014. 
[5] Th. Kecker, Polynomial Hamiltonian systems with movable algebraic singularities, arXiv:1312.4030.

[6] S. Shimomura, Growth of the first, the second and the fourth Painlevé transcendents, Math. Proc. Camb. Phil. Soc. 134 (2003), 259-269.

[7] N. Steinmetz, Zur Wertverteilung der Lösungen der vierten Painlevéschen Differentialgleichung, Math. Z. 181 (1982), 554-561.

[8] N. Steinmetz, Value distribution of the Painlevé transcendents, Israel J. Math. 128 (2002), 29-52.

[9] N. Steinmetz, The Yosida class is universal, Journal d'Analyse Math. 117 (2012), 347-364.

[10] N. Steinmetz, Complex Riccati differential equations revisited, Ann. Acad. Sci. Fenn. 39 (2014), 503-511.

[11] W. Wasow, Asymptotic expansions for ordinary differential equations, J. Wiley \& Sons 1965.

[12] K. Yosida, On a class of meromorphic functions, Proc. Phys. Math. Soc. Japan 16 (1934), $227-235$.

Norbert Steinmetz, Fakultät für Mathematik, Technische Universität Dortmund

stein@math.tu-dortmund.de, http://www.mathematik.tu-dortmund.de/steinmetz/ 